\title{
The thermodynamic instabilities of a binary mixture of sticky hard spheres
}

\author{
Riccardo Fantoni*, Domenico Gazzillo ${ }^{\dagger}$, and Achille Giacometti ${ }^{\ddagger}$ \\ Istituto Nazionale per la Fisica della Materia and Dipartimento di Chimica Fisica, \\ Università di Venezia, S. Marta DD 2137, I-30123 Venezia, Italy
}

(Dated: November 20, 2018)

The thermodynamic instabilities of a binary mixture of sticky hard spheres (SHS) in the modified Mean Spherical Approximation (mMSA) and the Percus-Yevick (PY) approximation are investigated using an approach devised by X. S. Chen and F. Forstmann [J. Chem. Phys. 97, 3696 (1992)]. This scheme hinges on a diagonalization of the matrix of second functional derivatives of the grand canonical potential with respect to the particle density fluctuations. The zeroes of the smallest eigenvalue and the direction of the relative eigenvector characterize the instability uniquely. We explicitly compute three different classes of examples. For a symmetrical binary mixture, analytical calculations, both for mMSA and for PY, predict that when the strength of adhesiveness between like particles is smaller than the one between unlike particles, only a pure condensation spinodal exists; in the opposite regime, a pure demixing spinodal appears at high densities. We then compare the mMSA and PY results for a mixture where like particles interact as hard spheres (HS) and unlike particles as SHS, and for a mixture of HS in a SHS fluid. In these cases, even though the mMSA and PY spinodals are quantitatively and qualitatively very different from each other, we prove that they have the same kind of instabilities. Finally, we study the mMSA solution for five different mixtures obtained by setting the stickiness parameters equal to five different functions of the hard sphere diameters. We find that four of the five mixtures exhibit very different type of instabilities. Our results are expected to provide a further step toward a more thoughtful application of SHS models to colloidal fluids.

\footnotetext{
* e-mail: rfantoni@unive.it

$\dagger$ e-mail: gazzillo@unive.it

$\ddagger$ e-mail: achille@unive.it
} 
PACS numbers: 64.60.-i, 64.70.-p, 64.70.Fx, 64.60.Ak

Keywords: Sticky Hard Spheres, modified Mean Spherical Approximation, Percus-Yevick, spinodal, demixing, condensation

\section{INTRODUCTION}

Thermodynamic instabilities are important to locate on the phase diagram of a fluid system those regions where the system can not exist as a single phase.

For a one-component system with Helmholtz free energy $A$, pressure $P$, in a volume $V$, at a temperature $T$, the condition for phase stability is $\left(\partial^{2} A / \partial V^{2}\right)_{T, N}=-(\partial P / \partial V)_{T, N}=$ $1 /\left(V \chi_{T}\right)>0$. The points where the isothermal compressibility $\chi_{T}$ diverges define the so called spinodal line, or phase instability boundary [1], that separates the stable from the unstable region of the phase diagram. In the stable region, where $\chi_{T}>0$, the system can exist in a single phase, while inside the other region the free energy can be lowered by phase separation into two phases with different densities. This kind of instability is usually referred to as mechanical instability, associated with a gas-liquid transition or condensation 1, 2, 3, 4].

In a binary mixture the situation is more complex 1, 2, 3, 4, 4, 5, 6]. The thermodynamic instability is located on the points of the phase diagram where $\left(\partial^{2} G / \partial x^{2}\right)_{T, P, N} / \chi_{T}=0$, where $x$ is the concentration of one of the two species, and $G$ is the Gibbs free energy. The points where $\chi_{T}^{-1}=0$ are instabilities of pure condensation (and the Bhatia Thornton [7] densitydensity structure factor, $S_{\rho \rho}(k)$, diverges at $\left.k=0\right)$. The points where $\left(\partial^{2} G / \partial x^{2}\right)_{T, P, N}=0$ are again instabilities of pure condensation when $\delta=\rho\left(v_{1}-v_{2}\right)=(\partial V / \partial x)_{T, P, N} / V$ diverges ( $\rho$ is the total number density, $v_{i}$ the partial molar volume, per particle, of species $i$. In this case all Bhatia Thornton structure factors diverge at $k=0$ ) and are instabilities of pure demixing when $\delta=0$ (in this case the Bhatia Thornton concentration-concentration structure factor, $S_{x x}(k)$ diverges at $k=0$ ). But, in general (for an asymmetric mixture), the kind of instability may be in between one of pure condensation and one of pure demixing, with $\delta$ finite and different from zero (also in this case all Bhatia Thornton structure factors diverge at $k=0)$. For the particular case of a binary symmetric mixture the only allowed instabilities are the ones of pure condensation and of pure demixing, since $\delta=0$.

A different route was followed by Chen and Forstmann [5] to characterize the instability 
uniquely in terms of an angle $\alpha$, function of the density and $x$.

The purpose of this work is to investigate the nature of instabilities for a binary mixture of sticky hard spheres (SHS). The SHS one-component model was originally proposed by Baxter [8, 9, 10], who showed how it admitted an analytic solution in the Percus-Yevick (PY) approximation. The PY solution was later extended to mixtures 11, 12, 13, 14] and it is nowaday regarded as extremely useful in colloidal systems. In the SHS model one accounts for a very short range attractive potential by defining an infinitely narrow and deep square well. This limit is carried out in a suitable way so that the second virial coefficient is finite. Due to its highly idealized nature, the one-component SHS model is not free of pathologies [15]. Nonetheless this model has recently regained considerable attention in studies of colloidal suspensions [16, 17, 18, 19] especially in its polydisperse version. Since the PY solution of a $p$-component SHS mixture requires the solution of $p(p+1) / 2$ coupled quadratic equations which are hard to solve for high $p$, attempts have been made to treat the model with "simpler" approximations [20, 21], which would allow analytic solution even for polydisperse systems. One of these approximations, that we will consider in this work, is the modified Mean Spherical Approximation (mMSA) 22].

In the present work we apply the Chen and Forstmann formalism to a binary SHS mixture, using both the mMSA and the PY approximation. The former can be regarded as the zero density limit of the latter and hence its predictions must be accepted with care. However it has its main merit in the fact that it entitles analytical predictions even in the multicomponent case, unlike the PY closure.

Three classes of systems will be discussed in details. First we consider the symmetric mixture, where equal-size equimolar components interact with variable strength only in the unlike part. This simplified case was already studied by Chen and Forstmann for hard core particles with attractive Yukawa interactions within the reference hypernetted chain approximation. In this particular SHS case we are able to perform a full characterization of the mixture both for mMSA and PY. In a second class we discuss two paradigmatic cases: (i) A fluid having HS interactions among like particles and SHS interactions for the unlike (System A) and (ii) A fluid formed by one SHS species and another HS one (System B). For PY both cases have been previously discussed by Barboy and Tenne 14], by Penders and Vrij [23], and by Regnaut, Amokrane, and Heno 24, 25] without, however, tackling the issue of the stability nature. Even in these two cases a detailed analytical investigation can be 
carried out. Building upon our recent work [26], we finally discuss a third class of examples involving a general binary mixture where, however, the stickiness parameters are related to the sizes of the particles according to some plausible prescriptions [26]. Within the mMSA, we are then able to discuss the nature of the instabilities previously calculated in Ref. [26], by evaluating numerically the Chen and Forstmann angle $\alpha$.

The remaining of the paper is organized as follows. In Section [1] we briefly outline Chen and Forstmann's approach, in Section III we report the PY and mMSA solutions for the Baxter factor correlation function of the SHS mixture. Section $\amalg$ is dedicated to the binary

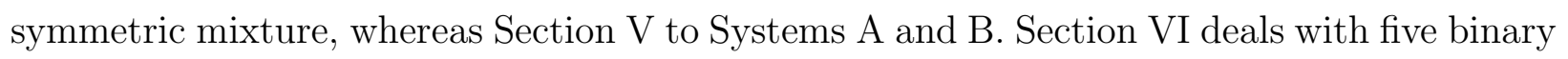
mixtures obtained setting the stickiness parameters equal to five different functions of the sphere diameters.

\section{METHOD FOR ANALYZING THE INSTABILITY}

For the sake of completeness, we briefly recall the main steps of the method reported in Ref. [5]. In doing this, however, we shall follow the general density functional formalism outlined in Ref. [27] which yields a clearer viewpoint.

\section{A. The Chen and Forstmann formalism}

Consider a binary mixture with $N_{1}$ particles of species 1 with coordinates $\mathbf{r}_{1}^{1}, \ldots, \mathbf{r}_{N_{1}}^{1}$ and $N_{2}$ particles of species 2 with coordinates $\mathbf{r}_{1}^{2}, \ldots, \mathbf{r}_{N_{2}}^{2}$ interacting through spherically symmetric pair potentials. Define the microscopic densities to be

$$
\boldsymbol{\rho}_{i}(\mathbf{r}) \equiv \sum_{\nu=1}^{N_{i}} \delta\left(\mathbf{r}-\mathbf{r}_{\nu}^{i}\right) \quad i=1,2
$$

for each one of the two species.

Consider now the non-homogeneous system with an external potential $\phi_{1}(\mathbf{r})$ acting on the particles of species 1 and an external potential $\phi_{2}(\mathbf{r})$ acting on the particles of species 2 . Let $\mu_{i}$ and $\Lambda_{i}$ be the chemical potential and the de Broglie thermal wavelength, respectively, for species $i, N=N_{1}+N_{2}$ the total number of particles, and $\mathbf{r}^{N}=\left(\left\{\mathbf{r}_{\nu}^{1}\right\},\left\{\mathbf{r}_{\nu}^{2}\right\}\right)$ a short-hand notation for the total set of coordinates. The grand partition function of the system with 
total internal energy $W\left(\mathbf{r}^{N}\right)$ is a functional of the generalized potentials $u_{i}(\mathbf{r})=\beta\left[\mu_{i}-\phi_{i}(\mathbf{r})\right]$

$$
\begin{aligned}
\Theta\left[u_{1}, u_{2}\right] & \equiv \sum_{N_{1}=0}^{\infty} \sum_{N_{2}=0}^{\infty} \frac{1}{\Lambda_{1}^{3 N_{1}} N_{1} !} \frac{1}{\Lambda_{2}^{3 N_{2}} N_{2} !} \int e^{-\beta W\left(\mathbf{r}^{N}\right)+\sum_{i=1}^{2} \int u_{i}(\mathbf{r}) \boldsymbol{\rho}_{i}(\mathbf{r}) d \mathbf{r}} d \mathbf{r}^{N} \\
& =e^{-\beta \Omega\left[u_{1}, u_{2}\right]}
\end{aligned}
$$

where $\Omega$ is the grand free energy. It can be proven [27] that the functional $\Omega$ is strictly concave in $u_{1}$ and $u_{2}$ (if we opportunely restrict its domain of definition). The equilibrium number density of species $i$ is given by

$$
\rho_{i}(\mathbf{r}) \equiv\left\langle\boldsymbol{\rho}_{i}(\mathbf{r})\right\rangle=-\frac{\delta \beta \Omega\left[u_{1}, u_{2}\right]}{\delta u_{i}(\mathbf{r})} .
$$

It follows that the following functional of $\left\{\rho_{i}\right\}$ and $\left\{u_{i}\right\}$

$$
\beta A\left[\rho_{1}, \rho_{2}, u_{1}, u_{2}\right] \equiv \sum_{i=1}^{2} \int \rho_{i}(\mathbf{r}) u_{i}(\mathbf{r}) d \mathbf{r}+\beta \Omega\left[u_{1}, u_{2}\right],
$$

is also strictly concave in $u_{1}$ and $u_{2}$, so it admits a unique maximum for $u_{i}=\bar{u}_{i}, i=1,2$, where the $\left\{\bar{u}_{i}\right\}$ can be determined univocally from Eq. (3) once the equilibrium densities $\left\{\rho_{i}\right\}$ are known.

We now set $\bar{A}\left[\rho_{1}, \rho_{2}\right] \equiv A\left[\rho_{1}, \rho_{2}, \bar{u}_{1}, \bar{u}_{2}\right]$. Again one can prove [27] that this Helmholtz free energy is a strictly convex functional in $\rho_{1}$ and $\rho_{2}$.

Introduce the following "grand free energy functional" of the densities

$$
\beta \Omega^{\prime}\left[\rho_{1}, \rho_{2}\right] \equiv \beta \bar{A}\left[\rho_{1}, \rho_{2}\right]-\sum_{i=1}^{2} \int \rho_{i}(\mathbf{r}) v_{i}(\mathbf{r}) d \mathbf{r},
$$

where $\left\{v_{i}\right\}$ are some given generalized potentials, independent of the densities. Clearly only when $v_{i}=\bar{u}_{i}, i=1,2$, we have $\Omega^{\prime}=\Omega$, i.e. equilibrium.

Taking the first functional derivative of $\Omega^{\prime}$ with respect to the densities we find

$$
\begin{aligned}
\frac{\delta \beta \Omega^{\prime}\left[\rho_{1}, \rho_{2}\right]}{\delta \rho_{i}(\mathbf{r})} & =\frac{\delta \beta \bar{A}\left[\rho_{1}, \rho_{2}\right]}{\delta \rho_{i}(\mathbf{r})}-v_{i}(\mathbf{r}) \\
& =\bar{u}_{i}(\mathbf{r})-v_{i}(\mathbf{r}),
\end{aligned}
$$

where in the second equality Eqs. (3) and (4) where used. At equilibrium we then have that the first functional derivatives of $\Omega^{\prime}$ vanish and $\Omega^{\prime}$ attains its minimum value.

The second functional derivatives of $\Omega^{\prime}$ with respect to the densities at equilibrium are 27]

$$
\left.\frac{\delta^{2} \beta \Omega^{\prime}\left[\rho_{1}, \rho_{2}\right]}{\delta \rho_{i}\left(\mathbf{r}_{1}\right) \delta \rho_{j}\left(\mathbf{r}_{2}\right)}\right|_{\text {equil. }}=\left.\frac{\delta \bar{u}_{i}\left(\mathbf{r}_{1}\right)}{\delta \rho_{j}\left(\mathbf{r}_{2}\right)}\right|_{\text {equil. }}=\frac{\delta_{i j} \delta\left(\mathbf{r}_{1}-\mathbf{r}_{2}\right)}{\rho_{i}\left(\mathbf{r}_{1}\right)}-c_{i j}\left(\mathbf{r}_{1}, \mathbf{r}_{2}\right)
$$


where $c_{i j}\left(\mathbf{r}_{1}, \mathbf{r}_{2}\right)$ are the partial direct correlation functions of the system.

So a Taylor expansion, up to the second order terms, yields the fluctuation of $\Omega^{\prime}$ around the equilibrium caused by small density fluctuations

$$
\begin{aligned}
\delta \Omega^{\prime} & =\Omega^{\prime}\left[\rho_{1}+\delta \rho_{1}, \rho_{2}+\delta \rho_{2}\right]-\Omega^{\prime}\left[\rho_{1}, \rho_{2}\right] \\
& =\frac{1}{2 \beta} \iint \sum_{i, j}\left[\frac{\delta_{i j} \delta\left(\mathbf{r}_{1}-\mathbf{r}_{2}\right)}{\rho_{i}\left(\mathbf{r}_{1}\right)}-c_{i j}\left(\mathbf{r}_{1}, \mathbf{r}_{2}\right)\right] \delta \rho_{i}\left(\mathbf{r}_{1}\right) \delta \rho_{j}\left(\mathbf{r}_{2}\right) d \mathbf{r}_{1} d \mathbf{r}_{2}
\end{aligned}
$$

If the system is homogeneous and isotropic at equilibrium (i.e. $\bar{u}_{i}(\mathbf{r})=\beta \mu_{i}, i=1,2$ ), so that

$$
\begin{aligned}
\rho_{i}(\mathbf{r}) & =\frac{N_{i}}{V}=\rho_{i} \\
c_{i j}\left(\mathbf{r}_{1}, \mathbf{r}_{2}\right) & =c_{i j}\left(\left|\mathbf{r}_{1}-\mathbf{r}_{2}\right|\right),
\end{aligned}
$$

where $V$ is the volume (assumed large enough), then we can rewrite the integral of Eq. (8), which is a convolution, as a k-integral of a product of Fourier transforms. Replacing the $\mathbf{k}$-integral $\left[(2 \pi)^{-3} \int d \mathbf{k} \ldots\right]$ by a sum over discrete $\mathbf{k}$-values $\left[V^{-1} \sum_{\mathbf{k}} \ldots\right]$, one obtains

$$
\delta \Omega^{\prime}=\frac{1}{2 \beta} \frac{1}{V} \sum_{\mathbf{k}} \sum_{i, j} \delta \bar{\rho}_{i}^{\star}(\mathbf{k}) \tilde{A}_{i j}(k) \delta \bar{\rho}_{j}(\mathbf{k}),
$$

where $\delta \bar{\rho}_{i}(\mathbf{k})=\delta \tilde{\rho}_{i}(\mathbf{k}) / \sqrt{\rho_{i}}$ and the asterisk indicates complex conjugation, having denoted with the tilde the Fourier transform

$$
\tilde{f}(\mathbf{k}) \equiv \int_{V} f(\mathbf{r}) e^{i \mathbf{k} \cdot \mathbf{r}} d \mathbf{r}
$$

so that

$$
\tilde{A}_{i j}(k)=\delta_{i j}-\sqrt{\rho_{i} \rho_{j}} \tilde{c}_{i j}(k)
$$

Notice that, due to the symmetry of the direct correlation functions under exchange of species indexes, the matrix $\tilde{\mathbf{A}}(k)$ is symmetric.

The probability distribution for the density fluctuations $\delta \rho_{i}$ (at constant $T, V$, and $\left\{\mu_{i}\right\}$ ) is proportional to $e^{-\beta \delta \Omega^{\prime}}[28]$. We therefore get for the mean values of the fluctuation products

$$
\begin{aligned}
\left\langle\delta \bar{\rho}_{i}^{\star}(\mathbf{k}) \delta \bar{\rho}_{j}(\mathbf{k})\right\rangle & =V\left[\tilde{\mathbf{A}}^{-1}\right]_{i j}(k) \\
& =V\left[\delta_{i j}+\sqrt{\rho_{i} \rho_{j}} \tilde{h}_{i j}(k)\right],
\end{aligned}
$$


where the last equality exploits the Ornstein-Zernike (OZ) equations between the partial total correlation functions $h_{i j}$ and the partial direct correlation functions.

Next define the molar fraction of species $i$ to be $x_{i}=\rho_{i} / \rho$, with $\rho=\sum_{i} \rho_{i}$ being the total density of the mixture. One usually introduces [7] two linear combinations of fluctuations of partial densities, i.e. the fluctuation of total density, $\delta \tilde{\rho}(\mathbf{k})$, and the fluctuation of concentration of species $1, \delta \tilde{x}(\mathbf{k})$,

$$
\begin{aligned}
\delta \tilde{\rho}(\mathbf{k}) & =\delta \tilde{\rho}_{1}(\mathbf{k})+\delta \tilde{\rho}_{2}(\mathbf{k}) \\
& =\sqrt{\rho}\left[\sqrt{x_{1}} \delta \bar{\rho}_{1}(\mathbf{k})+\sqrt{x_{2}} \delta \bar{\rho}_{2}(\mathbf{k})\right], \\
\delta \tilde{x}(\mathbf{k}) & =\frac{1}{\rho^{2}}\left[\rho_{2} \delta \tilde{\rho}_{1}(\mathbf{k})-\rho_{1} \delta \tilde{\rho}_{2}(\mathbf{k})\right] \\
& =\sqrt{\frac{x_{1} x_{2}}{\rho}}\left[\sqrt{x_{2}} \delta \bar{\rho}_{1}(\mathbf{k})-\sqrt{x_{1}} \delta \bar{\rho}_{2}(\mathbf{k})\right],
\end{aligned}
$$

so that, if $\delta \tilde{\rho}_{1}$ and $\delta \tilde{\rho}_{2}$ change in proportion to their respective mean concentration, then $\delta \tilde{x}=0$.

We also introduce

$$
\begin{aligned}
& \delta \bar{\rho}(\mathbf{k})=\frac{1}{\sqrt{\rho}} \delta \tilde{\rho}(\mathbf{k}), \\
& \delta \bar{x}(\mathbf{k})=\sqrt{\frac{\rho}{x_{1} x_{2}}} \delta \tilde{x}(\mathbf{k}),
\end{aligned}
$$

so that, in terms of the following two column vectors

$$
\mathbf{u}(\mathbf{k})=\left(\begin{array}{c}
\delta \bar{\rho}_{1}(\mathbf{k}) \\
\delta \bar{\rho}_{2}(\mathbf{k})
\end{array}\right), \quad \mathbf{v}(\mathbf{k})=\left(\begin{array}{c}
\delta \bar{\rho}(\mathbf{k}) \\
\delta \bar{x}(\mathbf{k})
\end{array}\right),
$$

Eqs. (15) and (16) can be written in compact notation as $\mathbf{u}=\mathbf{U v}$ where

$$
\mathbf{U}=\left(\begin{array}{cc}
\sqrt{x_{1}} & \sqrt{x_{2}} \\
\sqrt{x_{2}} & -\sqrt{x_{1}}
\end{array}\right),
$$

notice that $\mathbf{U}^{2}=\mathbf{I}$, where $\mathbf{I}$ is the identity matrix.

We find then from Eq. (111) (superscript $T$ indicating the transpose)

$$
\delta \Omega^{\prime}=\frac{1}{2 \beta} \frac{1}{V} \sum_{\mathbf{k}} \mathbf{v}^{T \star}(\mathbf{k}) \mathbf{M}(k) \mathbf{v}(\mathbf{k}),
$$

where $\mathbf{M}(k)$ is the following symmetric matrix

$$
\mathbf{M}(k)=\mathbf{U} \tilde{\mathbf{A}}(k) \mathbf{U}=\left(\begin{array}{ll}
M_{\rho \rho} & M_{\rho x} \\
M_{x \rho} & M_{x x}
\end{array}\right),
$$


with

$$
\begin{aligned}
& M_{\rho \rho}=1-\rho\left[x_{1}^{2} \tilde{c}_{11}+x_{2}^{2} \tilde{c}_{22}+2 x_{1} x_{2} \tilde{c}_{12}\right] \\
& M_{x x}=1-\rho x_{1} x_{2}\left[\tilde{c}_{11}+\tilde{c}_{22}-2 \tilde{c}_{12}\right] \\
& M_{\rho x}=M_{x \rho}=\rho \sqrt{x_{1} x_{2}}\left[x_{2} \tilde{c}_{22}-x_{1} \tilde{c}_{11}-\left(x_{2}-x_{1}\right) \tilde{c}_{12}\right] .
\end{aligned}
$$

The elements of the $\mathbf{M}(0)$ matrix are related to thermodynamic quantities [5] , as shown in the Appendix. In particular the determinant of $\mathbf{M}$ is

$$
\operatorname{det}(\mathbf{M})=x_{1} x_{2} \frac{\left(\chi_{T}^{0}\right)^{2}}{\chi_{T} V}\left(\frac{\partial^{2} G}{\partial x_{1}^{2}}\right)_{T, P, N}
$$

where $\chi_{T}$ is the isothermal compressibility, and $\chi_{T}^{0}=\beta / \rho$ is the isothermal compressibility of the ideal gas.

For the particular systems that we shall consider in the following, it turns out that the matrix $\tilde{\mathbf{A}}$ can be written, using the Wiener-Hopf factorization in terms of the Baxter factor matrix $\widehat{\mathbf{Q}}[11]$

$$
\tilde{\mathbf{A}}(k)=\widehat{\mathbf{Q}}^{T \star}(k) \widehat{\mathbf{Q}}(k) .
$$

Hence $\operatorname{det}[\mathbf{M}(k)]=\operatorname{det}[\tilde{\mathbf{A}}(k)]=|\operatorname{det}[\widehat{\mathbf{Q}}(k)]|^{2} \geq 0$ and $\operatorname{trace}[\mathbf{M}(k)]=\operatorname{trace}[\tilde{\mathbf{A}}(k)] \geq 0$.

The inverse of $\mathbf{M}(k)$ yields the mean square fluctuations of total density and concentration, i.e. the density-density structure factor $S_{\rho \rho}(k)$, the concentration-concentration structure factor $S_{x x}(k)$, and the cross term $S_{\rho x}(k)[7]$

$$
\begin{aligned}
& S_{\rho \rho}(k)=\frac{1}{V}\left\langle\delta \bar{\rho}^{\star}(\mathbf{k}) \delta \bar{\rho}(\mathbf{k})\right\rangle=\left[\mathbf{M}^{-1}\right]_{\rho \rho}(k), \\
& S_{x x}(k)=\frac{x_{1} x_{2}}{V}\left\langle\delta \bar{x}^{\star}(\mathbf{k}) \delta \bar{x}(\mathbf{k})\right\rangle=x_{1} x_{2}\left[\mathbf{M}^{-1}\right]_{x x}(k), \\
& S_{\rho x}(k)=\frac{\sqrt{x_{1} x_{2}}}{V}\left\langle\delta \bar{\rho}^{\star}(\mathbf{k}) \delta \bar{x}(\mathbf{k})\right\rangle=\sqrt{x_{1} x_{2}}\left[\mathbf{M}^{-1}\right]_{\rho x}(k) .
\end{aligned}
$$

Now, since $\mathbf{M}(k)$ is a symmetric matrix, it can be diagonalized through an orthogonal change of basis and it will have real eigenvalues

$$
\lambda_{ \pm}(k)=\frac{\operatorname{trace}[\mathbf{M}(k)] \pm \sqrt{\{\operatorname{trace}[\mathbf{M}(k)]\}^{2}-4 \operatorname{det}[\mathbf{M}(k)]}}{2}
$$

with $\lambda_{+}(k) \geq \lambda_{-}(k) \geq 0$. For the normalized eigenvectors we find

$$
\mathbf{z}_{ \pm}(k)=\left(\begin{array}{c}
a_{ \pm}(k) \\
b_{ \pm}(k)
\end{array}\right)
$$


with

$$
\begin{aligned}
& a_{ \pm}(k)=1 / \sqrt{1+\left(\frac{M_{\rho \rho}(k)-\lambda_{ \pm}(k)}{M_{\rho x}(k)}\right)^{2}}, \\
& b_{ \pm}(k)=-a_{ \pm} \frac{M_{\rho \rho}(k)-\lambda_{ \pm}(k)}{M_{\rho x}(k)} .
\end{aligned}
$$

The transition matrix to the base formed by the eigenvectors will be

$$
\mathbf{Z}(k)=\left(\begin{array}{cc}
a_{+}(k) & a_{-}(k) \\
b_{+}(k) & b_{-}(k)
\end{array}\right),
$$

Eq. (21) can then be recast into the form

$$
\delta \Omega^{\prime}\left(\delta \rho_{1}, \delta \rho_{2}\right)=\frac{1}{2 \beta} \frac{1}{V} \sum_{\mathbf{k}}\left[\lambda_{+}(k)\left|\delta \bar{\rho}_{+}(\mathbf{k})\right|^{2}+\lambda_{-}(k)\left|\delta \bar{\rho}_{-}(\mathbf{k})\right|^{2}\right],
$$

where $\delta \bar{\rho}_{ \pm}$are the Fourier components of the vector for the total density and concentration fluctuation in the eigenvector base, namely

$$
\mathbf{Z}^{-1} \mathbf{v}=\left(\begin{array}{c}
\delta \bar{\rho}_{+} \\
\delta \bar{\rho}_{-}
\end{array}\right),
$$

or

$$
\begin{aligned}
& \delta \bar{\rho}_{+}(\mathbf{k})=a_{+}(k) \delta \bar{\rho}(\mathbf{k})+b_{+}(k) \delta \bar{x}(\mathbf{k}), \\
& \delta \bar{\rho}_{-}(\mathbf{k})=a_{-}(k) \delta \bar{\rho}(\mathbf{k})+b_{-}(k) \delta \bar{x}(\mathbf{k}) .
\end{aligned}
$$

\section{B. Characterization of the instability}

We wish to know which combination of density and concentration fluctuations, $(\delta \bar{\rho}, \delta \bar{x})$ or $\left(\delta \bar{\rho}_{+}, \delta \bar{\rho}_{-}\right)$, yields the smallest increase $\delta \Omega^{\prime}$ of grand free energy. The border of a stability region (spinodal line) will be determined by the smaller eigenvalue $\lambda_{-}(k)$ going to zero. It is important to remark that the minimum eigenvalue will vanish if and only if $\operatorname{det}[\mathbf{M}(k)]=$ $\lambda_{-}(k) \lambda_{+}(k)=|\operatorname{det}[\widehat{\mathbf{Q}}(k)]|^{2}$ vanishes. The spinodal equation thus corresponds to

$$
\lambda_{-}(k)=0 \quad \text { or } \quad \operatorname{det}[\widehat{\mathbf{Q}}(k)]=0 .
$$

For all $\overline{\mathbf{k}}$-vectors with $\bar{k}=|\overline{\mathbf{k}}|$ being a solution of the spinodal equation, we can calculate the related eigenvector $\mathbf{z}_{-}(\bar{k})$ and find, from Eq. (39), one non-zero linear combination $\delta \bar{\rho}_{-}(\overline{\mathbf{k}})$ of density and concentration fluctuations for which $\delta \Omega^{\prime}=0$. Thus 
$\mathbf{z}_{-}(\bar{k})=\left[a_{-}(\bar{k}), b_{-}(\bar{k})\right]^{T}$ characterizes the phase transition uniquely. On defining the angle (see Fig. 1)

$$
\alpha=\arctan \left(\frac{a_{-}}{b_{-}}\right)_{k=\bar{k}}=\arctan \left[\frac{\widehat{Q}_{12}(\bar{k}) \sqrt{x_{1}}-\widehat{Q}_{11}(\bar{k}) \sqrt{x_{2}}}{\widehat{Q}_{12}(\bar{k}) \sqrt{x_{2}}+\widehat{Q}_{11}(\bar{k}) \sqrt{x_{1}}}\right],
$$

the instability will be predominantly of the demixing type when $\alpha$ is close to 0 (i.e. only concentration fluctuations occur) and predominantly of the condensation type when $\alpha$ is close to $\pm \pi / 2$ (i.e. only density fluctuates at fixed concentration).

The same feature can be seen in real space. When $\lambda_{-}(\bar{k})=0$ and $\alpha=0\left(\Rightarrow a_{-}=0, b_{+}=\right.$ 0 , and therefore $\left.\delta \bar{\rho}_{+}=a_{+} \delta \bar{\rho}, \delta \bar{\rho}_{-}=b_{-} \delta \bar{x}\right)$, one can get $\delta \Omega^{\prime}=0$ only if $\delta \bar{\rho}_{+}(\overline{\mathbf{k}})=0$, which requires $\delta \widetilde{\rho}(\overline{\mathbf{k}})=0$, i.e. the fluctuations that do not increase the "grand free energy" can be expressed as

$$
\begin{aligned}
& \delta \rho_{1}(\mathbf{r})=\frac{1}{V} \sum_{\substack{\mathbf{k}=\bar{k} \\
|\mathbf{k}|=\bar{k}}} \delta \rho_{1}(\mathbf{k}) e^{-i \mathbf{k} \cdot \mathbf{r}} \\
& \delta \rho_{2}(\mathbf{r})=-\delta \rho_{1}(\mathbf{r})
\end{aligned}
$$

On the other hand, when $\lambda_{-}(\bar{k})=0$ and $\alpha= \pm \pi / 2\left(\Rightarrow \delta \bar{\rho}_{+}=b_{+} \delta \bar{x}, \delta \bar{\rho}_{-}=a_{-} \delta \bar{\rho}\right)$, $\delta \bar{\rho}_{+}(\overline{\mathbf{k}})=0$ now requires $\delta \widetilde{x}(\overline{\mathbf{k}})=0$, which corresponds to

$$
\delta \rho_{2}(\mathbf{r})=+\frac{\rho_{2}}{\rho_{1}} \delta \rho_{1}(\mathbf{r})
$$

Eq. (42) yields oscillating partial density fluctuations for species 1 on the spinodal line, whereas Eqs. (43) and (44) represent the two different behaviors of the species 2 in correspondence to the two extreme values of $\alpha$ (0 and $\pm \pi / 2$, respectively). For $\alpha=0$, the fluctuations of species 2 must be in opposition of phase compared to those of species 1 , [see Eq. (43)], and this can be clearly interpreted as related to spatial demixing. In the opposite case $(\alpha= \pm \pi / 2)$, Eq. (44) means that an increase of species 1 in some region drives an increase of species 2 in the same region, a clear indication of a condensation type of instability. When $\alpha$ varies from zero to $\pm \pi / 2$ the allowed fluctuations will continuously vary from the pure demixing to the pure condensation type.

For a class of approximations (closures) having the partial direct correlation functions vanishing beyond a finite range, it was proven in [11] that $\widehat{\mathbf{Q}}(k)$ is non-singular for any $k>0$, so we can limit our search for the zeroes of the minimum eigenvalue to the case $k=0$. Moreover, since $\lim _{k \rightarrow \infty} \operatorname{det}[\widehat{\mathbf{Q}}(k)]=1$ and $\operatorname{det}[\widehat{\mathbf{Q}}(k)]$ is a continuous function of $k$, 
we must also have $\operatorname{det}[\widehat{\mathbf{Q}}(k=0)]$ non-negative, otherwise $\operatorname{det}[\widehat{\mathbf{Q}}(k)]$ would vanish for some finite $k$. We can use this last condition to determine which regions of the phase diagram are unstable. We cannot in fact gather this information by just looking at the matrix $\tilde{\mathbf{A}}$, which is always positive definite when non-singular. In the following, whenever we omit the dependence from the wave-vector $k$, we shall refer to the case $k=0$.

\section{THE BINARY STICKY HARD SPHERE FLUID}

We consider the SHS mixture described in the introduction by the following square-well interaction potential between a sphere of species $i$ and one of species $j$ [8, 9, 13, 14]

$$
\beta \phi_{i j}(r)= \begin{cases}+\infty & 0<r<\sigma_{i j} \\ -\ln \left(\frac{1}{12 \tau_{i j}} \frac{R_{i j}}{R_{i j}-\sigma_{i j}}\right) & \sigma_{i j} \leq r \leq R_{i j} \\ 0 & r>R_{i j}\end{cases}
$$

where $\beta=1 /\left(k_{B} T\right)\left(k_{B}\right.$ being Boltzmann constant and $T$ the temperature), $\sigma_{i j}=\left(\sigma_{i}+\sigma_{j}\right) / 2$ ( $\sigma_{i}$ being the diameter of a sphere of species $i$ ), $R_{i j}-\sigma_{i j}$ denotes the well width, and the dimensionless parameter

$$
\frac{1}{\tau_{i j}}=\frac{\epsilon_{i j}}{\tau}=\frac{\nu_{i j}}{\tau^{*}} \geq 0
$$

measures the strength of surface adhesiveness or "stickiness" between particles of species $i$ and $j$. In (46), $\tau$ is an unspecified increasing function of $T$, and we introduced the dimensionless quantities $\nu_{i j}=\epsilon_{i j} / \epsilon_{11}$ and $\tau^{*}=\tau / \epsilon_{11}$. The next step which defines the SHS model consists in taking the "sticky" limit $\left\{R_{i j}\right\} \rightarrow\left\{\sigma_{i j}\right\}$. Notice that the logarithm in the initial square-well potential (45) is chosen so to have a simple expression for the Boltzmann factor, which reduces to a combination of an Heaviside step function and a Dirac delta function in the sticky limit.

Within a class of mixed closures for which the partial direct correlation functions $c_{i j}(r)$ after the sticky limit vanish beyond $\sigma_{i j}$ [generalized PY (GPY) approximation [21]], the model can be analytically solved for the Baxter factor correlation function

$$
\begin{gathered}
q_{i j}(r)=\left\{\begin{array}{lc}
\frac{1}{2} a_{i}\left(r-\sigma_{i j}\right)^{2}+\left(b_{i}+a_{i} \sigma_{i j}\right)\left(r-\sigma_{i j}\right)+K_{i j}, & L_{i j}=\left(\sigma_{i}-\sigma_{j}\right) / 2 \leq r \leq \sigma_{i j}, \\
0, & \text { elsewhere },
\end{array}\right. \\
a_{i}=\frac{1}{\Delta}+\frac{3 \xi_{2} \sigma_{i}}{\Delta^{2}}-\frac{12 \zeta_{i}}{\Delta}, \quad b_{i}=\left(\frac{1}{\Delta}-a_{i}\right) \frac{\sigma_{i}}{2},
\end{gathered}
$$




$$
\xi_{n}=\frac{\pi}{6} \sum_{i} \rho_{i} \sigma_{i}^{n}, \quad \zeta_{i}=\frac{\pi}{6} \sum_{m} \rho_{m} \sigma_{m} K_{i m}, \quad \Delta=1-\xi_{3}
$$

The Baxter factor matrix $\widehat{\mathbf{Q}}(k)$ first introduced in Eq. (27) is related to Baxter factor correlation function through

$$
\widehat{Q}_{i j}(k)=\delta_{i j}-2 \pi \sqrt{\rho_{i} \rho_{j}} \widehat{q}_{i j}(k)
$$

where $\widehat{q}_{i j}(k)$ is the one-dimensional Fourier transform of $q_{i j}(r)$. It can be expressed in terms of spherical Bessel functions of the zeroth and first order and its explicit expression can be found in Eq. (27) of Ref. [20], and will not be reproduced here.

The symmetric matrix $K_{i j}$ is given by

$$
K_{i j}=\frac{\sigma_{i j}^{2}}{12 \tau_{i j}} \bar{y}_{i j}
$$

where $\bar{y}_{i j}=y_{i j}\left(\sigma_{i j}^{+}\right)$are the contact values of the partial cavity functions. For this kind of system a more natural parameter to use in place of the total density $\rho=\sum_{i} \rho_{i}$ is the total packing fraction $\eta=\xi_{3}$.

In the modified Mean Spherical Approximation $\left[c_{i j}(r)=f_{i j}(r)\right.$ when $r>\sigma_{i j}$, where $f_{i j}(r)=\exp \left[-\beta \phi_{i j}(r)\right]-1$ are the Mayer functions] one can show [21] that 29]

$$
\bar{y}_{i j}=1 \text { for all } i \text { and } j
$$

In the Percus-Yevick approximation $\left[c_{i j}(r)=f_{i j}(r) y_{i j}(r)\right]$ one can show that the $\bar{y}_{i j}$ have to satisfy the following set of coupled quadratic equations [13]

$$
\bar{y}_{i j} \sigma_{i j}=a_{i} \sigma_{i j}+b_{i}+2 \pi \sum_{k} \rho_{k} \frac{\sigma_{k j}^{2}}{12 \tau_{k j}} \bar{y}_{k j} q_{k i}\left(L_{k i}\right)
$$

It is worth stressing that the above expressions are valid for both the mMSA and the PY closures, provided that the correct values of $\bar{y}_{i j}$ are inserted into the matrix $K_{i j}$ given in Eq. (51) 20, 26]. All the results gathered so far in this Section are valid for a generic $p$-component SHS mixture. In the rest of the work we will specialize to two-component $(p=2)$ mixtures. For a binary mixture the determinant of $\widehat{\mathbf{Q}}(0)$ can be reduced to the following simple expression [30]

$$
\begin{aligned}
\operatorname{det}[\widehat{\mathbf{Q}}(0)]= & \frac{1+2 \eta}{(1-\eta)^{2}}-\frac{\eta_{1} \lambda_{11}^{B T}+\eta_{2} \lambda_{22}^{B T}}{(1-\eta)^{2}}- \\
& \frac{\eta_{1} \eta_{2}}{(1-\eta)^{3}}\left[3\left(\lambda_{11}^{B T}+\lambda_{22}^{B T}-2 \lambda_{12}^{B T}\right)-\lambda_{11}^{B T} \lambda_{22}^{B T}+\left(\lambda_{12}^{B T}\right)^{2}\right],
\end{aligned}
$$


where

$$
\begin{aligned}
\eta_{i} & =\frac{\pi}{6} \rho_{i} \sigma_{i}^{3}, \\
\lambda_{i j} & =\frac{\bar{y}_{i j}}{\tau_{i j}}, \\
\lambda_{i j}^{B T} & =(1-\eta) \lambda_{i j} \frac{\sigma_{i j}^{2}}{\sigma_{i} \sigma_{j}} .
\end{aligned}
$$

Our task is the determination of the spinodal line and of the nature of the instability. These can be expressed respectively by the reduced temperature $\tau^{*}=f_{\tau}\left(\rho \sigma_{1}^{3}, x_{1}, \zeta,\left\{\nu_{i j}\right\}\right)$ and the angle $\alpha=f_{\alpha}\left(\rho \sigma_{1}^{3}, x_{1}, \zeta,\left\{\nu_{i j}\right\}\right)$, where $\zeta=\sigma_{2} / \sigma_{1}$. Sometimes it also proves convenient to use another set of independent variables, namely $\eta, x_{1}, \zeta,\left\{\nu_{i j}\right\}$.

We anticipate that, while $f_{\tau}$ will in general depend on the particular chosen closure, $f_{\alpha}$ need not mirror this feature. An example is the case studied in Section $\mathbf{V}$, where two $\epsilon_{i j}$ are zero and $\epsilon_{\bar{\imath} \bar{\jmath}}>0$. Then $\lambda_{i j}=0$ for $i \neq \bar{\imath}$ or $j \neq \bar{\jmath}$ and the spinodal equation

$$
\lambda_{-}(0)=0 \quad \text { or } \quad \operatorname{det}[\widehat{\mathbf{Q}}(0)]=0 .
$$

is sufficient for determining the third $\lambda$, which turns out to be a function $\lambda_{\bar{\imath} \bar{\jmath}}\left(\eta, x_{1}, \zeta\right)$ independent from the particular closure within the class we are considering. Since in each matrix element of $\widehat{\mathbf{Q}}$ the quantities $\bar{y}_{i j}$ and $\tau_{i j}$ appear only in the ratios $\lambda_{i j}$, it follows that the angle $\alpha$ [see Eq. (41)] will also be independent of the particular closure.

In the case of a general binary mixture (with two or three non-vanishing $\epsilon_{i j}$ ) we expect a dependence of the angle from the closure, even if this point would deserve further investigation.

\section{THE SYMMETRIC BINARY MIXTURE}

The PY approximation leads, even in the simple binary case, to the solution of two coupled quartic equations. We then start with a simpler task, akin to the one already discussed by Chen and Forstmann [5] for a different potential, of finding the spinodal line and angle $\alpha$ predicted by the mMSA and PY for the symmetric binary mixture. In this case $x_{1}=x_{2}=1 / 2, \sigma_{1}=\sigma_{2}=\sigma$, and $\epsilon_{11}=\epsilon_{22}$. By symmetry we must have $\tilde{c}_{11}=\tilde{c}_{22}$ and from Eq. (25) it follows that $\mathbf{M}$ is diagonal, the cross term $M_{\rho x}$ being identically zero and

$$
\lambda_{-}=\min \left\{M_{\rho \rho}, M_{x x}\right\}
$$


Therefore the symmetric mixture can only have either pure condensation $(\alpha= \pm \pi / 2)$ or pure demixing $(\alpha=0)$ instabilities.

Moreover for the symmetric mixture we have from Eqs. (28)-(30)

$$
\begin{aligned}
S_{\rho \rho} & =\frac{1}{M_{\rho \rho}}, \\
S_{x x} & =\frac{1}{4 M_{x x}}, \\
S_{\rho x} & =0 .
\end{aligned}
$$

We see then that on a pure condensation instability $S_{\rho \rho}(0) \rightarrow \infty$ or $\tilde{h}_{11}(0)+\tilde{h}_{12}(0) \rightarrow \infty$, whereas on a pure demixing instability $S_{x x}(0) \rightarrow \infty$ or $\tilde{h}_{11}(0)-\tilde{h}_{12}(0) \rightarrow \infty$, and each type of instability shows a distinct form of long-range behavior in the correlation functions.

\section{A. Symmetric mixture in the mMSA}

Let us first consider the symmetric mixture within the mMSA. The spinodal line will be of pure condensation when $M_{\rho \rho}=0$, that is

$$
\tau^{*}=\tau_{\rho}^{*}=\left(1+\nu_{12}\right) \frac{1}{2} \eta \frac{1-\eta}{1+2 \eta},
$$

whose maximum in the $\left(\tau^{*}, \eta\right)$ plane occurs at $\eta=\eta_{c}^{m M S A}=(\sqrt{3}-1) / 2=0.3660 \ldots$ (independently of $\nu_{12}$ ). On the other hand the spinodal will be a line of pure demixing when $M_{x x}=0$ which has as solution

$$
\tau^{*}=\tau_{x}^{*}=\left(1-\nu_{12}\right) \frac{1}{2} \eta
$$

Note that the allowed packing fractions are the ones smaller than the close packed packing fraction $\eta_{0}=\pi \sqrt{2} / 6=0.7404 \ldots$

For the determinant of $\widehat{\mathbf{Q}}(0)$ we find from Eq. (54)

$$
\operatorname{det}[\widehat{\mathbf{Q}}(0)]=\frac{\left(\tau-\tau_{\rho}\right)\left(\tau-\tau_{x}\right)}{\tau^{2}} \frac{1+2 \eta}{(1-\eta)^{2}}
$$

so that the system is unstable when $\tau$ lies between the two roots $\tau_{\rho}$ and $\tau_{x}$, at a given packing fraction.

While the condensation line is always present, the existence of a demixing line depends upon the value of $\nu_{12}$, as expected. When $\nu_{12} \geq 1$ the demixing line $\tau^{*}=\tau_{x}^{*}$ lies below the 
$\eta$-axis, and hence the spinodal in the phase diagram $\left(\tau^{*}, \eta\right)$ is the curve $\tau^{*}=\tau_{\rho}^{*}$ (see Fig. 22), with the instability being of pure condensation at all densities. Notice that this would be the case for Lorentz-Berthelot mixtures for which we have $\epsilon_{12} \equiv \sqrt{\epsilon_{11} \epsilon_{22}}=\epsilon_{11}$, which corresponds to $\nu_{12}=1$, that is the one-component case.

When $\nu_{12}<1$ the two roots $\tau^{*}=\tau_{\rho}^{*}$ and $\tau^{*}=\tau_{x}^{*}$ intercept at a point [31] (see Fig. 33) having packing fraction

$$
\eta=\eta_{\rho x}=\frac{2 \nu_{12}}{3-\nu_{12}}<1,
$$

so the instability is of pure condensation for $\eta<\eta_{\rho x}$ and of pure demixing for $\eta>\eta_{\rho x}$.

\section{B. Symmetric mixture in the PY}

In the PY approximation we first need to determine the cavity functions at contact. Eqs. (53) for the binary symmetric mixture can be recast into the following form

$$
\begin{aligned}
\lambda_{11} \tau_{11}-\frac{1}{2} \eta\left(\frac{1}{12} \lambda_{11}^{2}-\frac{1}{\Delta} \lambda_{11}\right) & =\bar{y}_{11}^{H S}+\frac{1}{2} \eta\left(\frac{1}{12} \lambda_{12}^{2}-\frac{1}{\Delta} \lambda_{12}\right), \\
\lambda_{12} \tau_{12}\left[1+\frac{1}{\tau_{12}}\left(\frac{\eta}{2 \Delta}-\frac{1}{12} \eta \lambda_{11}\right)\right] & =\bar{y}_{12}^{H S}-\frac{\eta}{2 \Delta} \lambda_{11},
\end{aligned}
$$

where

$$
\bar{y}_{11}^{H S}=\bar{y}_{12}^{H S}=\bar{y}^{H S}=\frac{2+\eta}{2(1-\eta)^{2}},
$$

is the HS expression for the cavity functions at contact. Substitution of Eq. (68) into Eq. (67) leads to a quartic equation for $\lambda_{11}$. The solution for the cavity functions at contact can then be written as

$$
\begin{aligned}
& \frac{\bar{y}_{11}}{\tau_{11}}=R, \\
& \frac{\bar{y}_{12}}{\tau_{12}}=\frac{\bar{y}^{H S}-\frac{\eta}{2 \Delta} R}{\tau_{12}\left[1+\frac{1}{\tau_{12}}\left(\frac{\eta}{2 \Delta}-\frac{1}{12} \eta R\right)\right]},
\end{aligned}
$$

where $R$ is a solution of the quartic equation.

In order to find the physically meaningful zeroes of $M_{\rho \rho}$ and $M_{x x}$ we proceed as follows. First we compute all the four roots $R_{i}, i=a, b, c, d$ of the quartic equation and hence $\left(\bar{y}_{11}\right)_{i}=\left(\bar{y}_{11}\right)_{i}\left(\tau^{*}, \eta, \nu_{12}\right)$, and $\left(\bar{y}_{12}\right)_{i}=\left(\bar{y}_{12}\right)_{i}\left(\tau^{*}, \eta, \nu_{12}\right)$ are the cavity functions at contact obtained using the root $R_{i}$, while $\left(M_{\rho \rho}\right)_{i}$ and $\left(M_{x x}\right)_{i}$ are the diagonal elements of $\mathbf{M}$ obtained using for the cavity functions at contact $\left(\bar{y}_{11}\right)_{i}$ and $\left(\bar{y}_{12}\right)_{i}$. As it turns out, only two roots 
$R_{i}$ will give physically admissible cavity functions at contact. Then we compute the zeroes of $\left(M_{\rho \rho}\right)_{i}$, denoted as $\tau^{*}=\left(\tau_{\rho}^{*}\right)_{i}\left(\eta, \nu_{12}\right)$, and of $\left(M_{x x}\right)_{i}$, denoted as $\tau^{*}=\left(\tau_{x}^{*}\right)_{i}\left(\eta, \nu_{12}\right)$. Then physical zeroes are then selected by the requirement that

$$
\lim _{\nu_{12} \rightarrow 1}\left(\bar{y}_{11}\right)_{i}\left(\left(\tau_{\alpha}^{*}\right)_{i}, \eta, \nu_{12}\right)=\lim _{\nu_{12} \rightarrow 1}\left(\bar{y}_{12}\right)_{i}\left(\left(\tau_{\alpha}^{*}\right)_{i}, \eta, \nu_{12}\right)=\bar{y}_{+}^{o c}\left(\left(\tau_{\alpha}^{*}\right)_{i}, \eta\right) \quad \alpha=\rho, x
$$

where $\bar{y}_{+}^{o c}$ is the physical cavity function at contact for the one-component system

$$
\bar{y}_{ \pm}^{o c}(\tau, \eta)=\frac{\bar{y}^{H S}}{\frac{1}{2}\left[1+\frac{\eta}{\Delta} \frac{1}{\tau} \pm \sqrt{\left(1+\frac{\eta}{\Delta} \frac{1}{\tau}\right)^{2}-\frac{\eta}{3} \bar{y}^{H S} \frac{1}{\tau^{2}}}\right]}
$$

Using constraint (72) we find that the correct physical solution is $R=R_{b}$ at high $\eta$, the only one such that

$$
\begin{aligned}
\lim _{\tau \rightarrow \infty}\left(\bar{y}_{11}\right)_{b} & =\lim _{\tau \rightarrow \infty}\left(\bar{y}_{12}\right)_{b}=\bar{y}^{H S}, \\
\lim _{\eta \rightarrow 0}\left(\bar{y}_{11}\right)_{b} & =\lim _{\eta \rightarrow 0}\left(\bar{y}_{12}\right)_{b}=1,
\end{aligned}
$$

while at small $\eta$ the solution to use is $R=R_{a}$ such that condition (72) is satisfied. As for the one-component system there is an interval $\left[0, \eta_{e}\right]$ where there are no physical zeroes. For the one-component case the spinodal

$$
\tau^{*}=\tau_{o c}^{*}=\frac{1+4 \eta-14 \eta^{2}}{12(1-\eta)(1+2 \eta)},
$$

exists only if $\eta>\eta_{e}$ where $\eta_{e}=\eta_{c}^{P Y}=(3 \sqrt{2}-4) / 2=0.1213 \ldots$ and $\eta_{c}^{P Y}$ is the PY critical packing fraction. For the binary symmetric mixture, numerical results strongly suggests the coincidence of $\eta_{e}$ with the critical packing fraction (see Figs. 4, 5, and 5) but we have not succeeded in proving it (nor in determining an expression for it). The unphysical continuation of the pure condensation spinodal in the range $\left[0, \eta_{e}\right]$ is given by the root $R=R_{c}$ such that

$$
\lim _{\nu_{12} \rightarrow 1}\left(\bar{y}_{11}\right)_{c}\left(\left(\tau_{\alpha}^{*}\right)_{c}, \eta, \nu_{12}\right)=\lim _{\nu_{12} \rightarrow 1}\left(\bar{y}_{12}\right)_{c}\left(\left(\tau_{\alpha}^{*}\right)_{c}, \eta, \nu_{12}\right)=\bar{y}_{-}^{o c}\left(\left(\tau_{\alpha}^{*}\right)_{c}, \eta\right) \quad \alpha=\rho, x
$$

Notice that this solution would also give, in the same range of $\eta$, an unphysical spinodal of pure demixing whenever $\nu_{12}<1$.

The zeroes $\tau^{*}=\left(\tau_{\rho}^{*}\right)_{i}$ and $\tau^{*}=\left(\tau_{x}^{*}\right)_{i}$ are shown in Fig. 4 for $\nu_{12}=2$, and for $\nu_{12}=2 / 3$ (the same conditions as in Figs. 22 and 3, respectively). As it happened in the mMSA, for $\nu_{12}>1$ there is only a spinodal of pure condensation, while for $\nu_{12}<1$ a spinodal of pure 
demixing appears at high $\eta$, as expected on physical grounds. However, unlike the mMSA case, the pure demixing and the pure condensation lines do not merge. Also the shapes and numerical values of the PY spinodals significantly differ from the mMSA ones. In Fig. 5 we select $\nu_{12}$ slightly above 1 and slightly below 1 in order to check the correct convergence towards the one-component case. At $\nu_{12}=1 / 1.1$ the line of pure demixing appears in the physically non-accessible region $\eta>\eta_{0}$.

\section{TWO PARADIGMATIC SYSTEMS}

The next two mixtures can be regarded as paradigmatic examples of a system where one expects to have a predominant condensation or predominant demixing type of thermodynamic instability. The thermodynamics of these mixtures have been previously investigated by Barboy and Tenne [14] within the PY approximation. In the following we shall extend this analysis of the instability type both within mMSA and PY closures. The two systems are defined as follows: (System A) $\epsilon_{12}>0, \epsilon_{11}=\epsilon_{22}=0$; (System B) $\epsilon_{11}>0, \epsilon_{12}=\epsilon_{22}=0$. System A corresponds to a fluid where the HS potential acts between like particles and the SHS potential between unlike particles, while System B corresponds to HS (species 2) in a SHS fluid (species 1). Alternatively, on regarding the large spheres as the "solute" and the small spheres as the "solvent", Systems A and B can be reckoned as a schematic model mimicking a "good" and a "poor" solvent, respectively [23].

For System A we have $\lambda_{11}^{B T}=\lambda_{22}^{B T}=0$, so Eq. (53) reduces to a a linear equation for $\bar{y}_{12}$ with the following solution (which corrects Eq. (64) of Barboy and Tenne)

$$
\bar{y}_{12}=\frac{\bar{y}_{12}^{H S}}{1+\frac{\xi_{2}}{2 \Delta} \frac{\sigma_{12}}{\tau_{12}}},
$$

where

$$
\bar{y}_{12}^{H S}=\frac{1}{\Delta}+\frac{3}{2} \frac{\xi_{2}}{\Delta^{2}} \frac{\sigma_{1} \sigma_{2}}{\sigma_{12}},
$$

is the HS expression for the contact cavity function.

For System B we have $\lambda_{12}^{B T}=\lambda_{22}^{B T}=0$, so Eq. (533) reduces to a quadratic equation for $\bar{y}_{11}$. The only solution which reduces to the HS expression for $\tau_{11} \rightarrow \infty$, is (identical to Eq. 
(57) of Barboy and Tenne)

$$
\bar{y}_{11}=\frac{\bar{y}_{11}^{H S}}{\frac{1}{2}\left[1+\frac{\eta_{1}}{\Delta} \frac{1}{\tau_{11}}+\sqrt{\left(1+\frac{\eta_{1}}{\Delta} \frac{1}{\tau_{11}}\right)^{2}-\frac{\eta_{1}}{3} \bar{y}_{11}^{H S} \frac{1}{\tau_{11}^{2}}}\right]}
$$

where

$$
\bar{y}_{11}^{H S}=\frac{1}{\Delta}+\frac{3}{2} \frac{\xi_{2}}{\Delta^{2}} \sigma_{1},
$$

is the HS expression for the contact cavity function. The instability lines are again given by Eq. (58).

Let $\lambda_{\bar{\imath} \bar{\jmath}}\left(\eta, x_{1}, \zeta\right)$ be the solution of the spinodal equation (58) for the only non-vanishing $\lambda_{i j}$. As the cavity functions must be positive, the spinodal exists only for those values of $\eta, x, \zeta$ for which $\lambda_{\bar{\imath} \bar{\jmath}}>0$. It may also happen (and it does in the PY case) that the spinodal equation

$$
\lambda_{\bar{\imath} \bar{\jmath}}^{(\text {closure })}=\frac{\bar{y}_{\bar{\imath} \bar{\jmath}}^{(\text {closure })}\left(\tau_{\bar{\imath} \bar{\jmath}}, \eta, x_{1}, \zeta\right)}{\tau_{\bar{\imath} \bar{\jmath}}}=\lambda_{\bar{\imath} \bar{\jmath}}\left(\eta, x_{1}, \zeta\right)
$$

upon choosing the correct physical solution for $\bar{y}_{\bar{\imath} \bar{\jmath}}^{(\text {closure })}$, does not have any real positive solutions for $\tau$, at certain values of $\eta, x, \zeta$. For these values the spinodal predicted by the particular closure has loss of solution and the predicted value for the angle $\alpha$ has clearly no physical meaning.

\section{A. Instabilities for System A}

On setting

$$
\lambda_{A}^{B T}=3+\sqrt{\left(3+\frac{\Delta}{\eta_{1}}\right)\left(3+\frac{\Delta}{\eta_{2}}\right)},
$$

for System A the solution of Eq. (58) within the mMSA approximation is

$$
\tau_{12}^{m M S A}=\frac{\Delta}{\lambda_{A}^{B T}} \frac{\sigma_{12}^{2}}{\sigma_{1} \sigma_{2}} .
$$

while in the PY is

$$
\tau_{12}^{P Y}=\bar{y}_{12}^{H S} \tau_{12}^{m M S A}-\frac{\xi_{2} \sigma_{12}}{2 \Delta}
$$

and, in the limit of high dilution while keeping $\tau_{12}^{m M S A}$ constant, one finds $\tau_{12}^{P Y} \rightarrow \tau_{12}^{m M S A}$, as expected in view of the fact that the PY contact cavity functions converge towards the mMSA contact cavity functions. 
In order to exist, the instability line must clearly lie on the $\tau_{12}>0$ side of the $\left(\tau_{12}, \eta\right)$ plane. It is easy to see that, while

$$
\left.\frac{d \tau_{12}^{m M S A}}{d \eta}\right|_{\eta=0}>0 \text { for any choice of } x_{1} \text { and } \zeta,
$$

we have that $\left(\sigma_{1}<\sigma_{2}\right)$

$$
\left.\frac{d \tau_{12}^{P Y}}{d \eta}\right|_{\eta=0}>0 \text { only when } \frac{\sigma_{2}}{\sigma_{1}+\sigma_{2}}<x_{1}<\frac{\sigma_{2}^{3}}{\sigma_{1}^{3}+\sigma_{2}^{3}} .
$$

So in the PY approximation the thermodynamic instability disappears as $x_{1}$ falls outside the range indicated in Eq. (87).

In Figs. 6] and 7 we depict the mMSA and PY spinodals, respectively, at a given value of $\zeta$ and three different values of $x_{1}$ for which the PY spinodal does exist. One clearly sees that conditions (86) and (87) result in a large scale difference between the two plots.

As regards the angle $\alpha$, we know, from the discussion at the end of Section III, that the angles predicted by the two approximations are the same, and from Fig. 8 we see that the kind of instability is mainly of condensation type in accord with what we expected from the outset. Two exact limits are worth mentioning. First, the infinite dilute limit

$$
\lim _{\eta \rightarrow 0} \alpha=\arctan \left(\frac{\sqrt{x_{2} / x_{1}}+\sqrt{\sigma_{2} / \sigma_{1}}}{\sqrt{\sigma_{2} x_{2} /\left(\sigma_{1} x_{1}\right)}-1}\right),
$$

provides an analytical check of the numerical results reported in Fig. 8. Second, when $\sigma_{2} \gg \sigma_{1}$ one obtains

$$
\lim _{\zeta \rightarrow \infty} \alpha=\arctan \sqrt{x_{1} / x_{2}}
$$

This result bears an interesting physical interpretation. As the fraction $x_{2}$ of large particles decreases, the angle $\alpha$ tends to $\pi / 2$, that is to a condensation instability. This is in striking contrast with what one would expect for HS on the basis of an entropic depletion mechanism [32, 33], which would tend to favor demixing in a system with a small number of large spheres. The reason for this can be traced back to the fact that in System A unlike particles have attractive interactions, thus preventing smaller particles to slip out from the interstitial region between two larger spheres. This interpretation also holds true if one regards system A as a good solvent. 


\section{B. Instabilities for System B}

Denoting

$$
\lambda_{B}^{B T}=\frac{(1+2 \eta)(1-\eta)}{\eta_{1}(1-\eta)+3 \eta_{1} \eta_{2}}
$$

for System B the solution of Eq. (58) within the mMSA is

$$
\tau_{11}^{m M S A}=\frac{\Delta}{\lambda_{B}^{B T}},
$$

while in the PY approximation is

$$
\tau_{11}^{P Y}=\bar{y}_{11}^{H S} \tau_{11}^{m M S A}+\frac{\eta_{1}}{12 \tau_{11}^{m M S A}}-\frac{\eta_{1}}{\Delta}
$$

for

$$
\tau_{11}^{P Y}>\frac{\eta_{1}}{\Delta}\left(\frac{\lambda_{B}^{B T}}{6}-1\right) .
$$

In view of the above constraint, there is an interval $\eta \in\left[0, \eta_{e}\right]$ where no physical spinodal exists. We stress that only for the one-component SHS limit $\left(x_{2}=0\right)$ one finds that $\eta_{e}=\eta_{c}$, with $\eta_{c}$ being the critical packing fraction, whereas in the more general case, studied here, this occurrence is no longer true, as shown in Fig. 10. Once again $\tau_{11}^{P Y}$, as given in Eq. (92), reduces to $\tau_{11}^{m M S A}$, in the limit of high dilution, with $\tau_{11}^{m M S A}$ kept constant. However, unlike $\tau_{11}^{m M S A}$, which is always a concave function of $\eta$ for any choice of $x_{1}$ and $\zeta, \tau_{11}^{P Y}$, it may display a van der Waals loop (see Fig. 10) as a function of $\eta$. The shape of the spinodal is strongly dependent on the content of the HS component in the mixture. When $x_{1}<\bar{x}_{1}$ $\left(\bar{x}_{1} \approx 0.8681 \ldots\right.$ when $\left.\zeta=1\right)$ the spinodal is a monotonously increasing function of $\eta$, while for $x_{1}>\bar{x}_{1}$ a loop appears. This point has already been emphasized by Barboy and Tenne [14].

As previously remarked, even in this case both mMSA and PY results for $\alpha$ coincide in the respective range of existence. In Fig. 11 we see that the instability for System B tends to pure demixing for $\zeta=1$ and large $\eta$. As $\zeta$ is increased, one finds the same limit (89) as

for System A. Once again the osmotic depletion mechanism fails because of the presence of stickiness this time among the small particles. As a further support to this interpretation, one also finds in the opposite limit

$$
\lim _{\zeta \rightarrow 0} \alpha=\arctan \left(\frac{x_{1}-\eta}{\sqrt{x_{1} x_{2}}}\right) .
$$


In this case, when $x_{1}=\eta$ the instability of the system is of a pure demixing type, so the solvent (particles of species 2) is a "poor" one. This is because the smaller particles (species 2) interact as HS both with larger spheres (species 1) and with each other. Hence, not only the depletion mechanism is not opposed in the present case, but, quite on the contrary, is favored by the attraction occurring between two big spheres (see Fig. 9). This results into the possibility for the existence of a demixing instability even if the HS binary mixture, within the closures considered here, does not have any instability [see Eq. (98)]. One can also show that

$$
\lim _{\eta \rightarrow 0} \alpha^{m M S A}=\arctan \sqrt{x_{1} / x_{2}},
$$

where $\alpha^{m M S A}$ is the angle predicted by the mMSA, whose spinodal does not have loss of solution at small $\eta$, or, upon using $\eta_{1}, \eta_{2}, y$ as independent variables, $\lim _{\eta_{1} \rightarrow 0} \alpha^{m M S A}=0$.

Before closing this section, a word of caution should be given on the aforementioned interpretations. In order to have a clear and quantitative understanding of the depletion mechanism discussed in this section (for both System A and B), the depletion potential, that is the effective potential among the large spheres mediated by the presence of the small ones, should be computed. Hence, the aforementioned scenarios should only be considered as a plausible possibility rather than a definite statement.

\section{FIVE BINARY MIXTURES TREATED WITH MMSA}

As a final point it is instructive to consider a more general example. To this aim, it proves convenient to relate the adhesion strengths $\epsilon_{i j}$ to the particle sizes $\left\{\sigma_{i}\right\}$. Our past

experience [26] suggests to consider five different cases, obtained setting $\epsilon_{i j} / \epsilon_{0}=\mathcal{F}_{i j}^{\mu}\left(\sigma_{1}, \sigma_{2}\right)$ for $\mu=1,2,3,4$, and 5. The functions $\mathcal{F}^{\mu}$ are selected as follows [26]

$$
\frac{\epsilon_{i j}}{\epsilon_{0}}= \begin{cases}\langle\sigma\rangle^{2} / \sigma_{i j}^{2} & \text { Case I } \\ \sigma_{i} \sigma_{j} / \sigma_{i j}^{2} & \text { Case II } \\ \left\langle\sigma^{2}\right\rangle / \sigma_{i j}^{2} & \text { Case III } \\ 1 & \text { Case IV } \\ \langle\sigma\rangle / \sigma_{i j} & \text { Case V }\end{cases}
$$

where $\langle F\rangle \equiv \sum_{i} x_{i} F_{i}$. A critical justification leading to the above choice can be found in Ref. [26]. Note that since for all five Cases the $\epsilon_{i j}$ are homogeneous functions of order zero in 
the diameters $\left\{\sigma_{i}\right\}$, the corresponding mixtures are invariant under a transformation where $V \rightarrow \lambda V$ and all $\sigma_{i} \rightarrow \lambda \sigma_{i}$ with $\lambda$ a scale factor [34].

We have calculated the angle $\alpha$ defined in Eq. (41) on the spinodal [Eq. 58] for all the Cases listed in (96) within the mMSA closure. The angle $\alpha$ turns out to be the same for Case I and III. The results are shown in Figs. 12 and 13 for $x_{1}=1 / 2$ and two different values of $\zeta$. We have only considered packing fractions $\eta \leq \eta_{m}=\pi \sqrt{2} / 6$, where $\eta_{m}$ is the maximum packing fraction for a "completely demixed" HS mixture (i.e. the packing fraction of a mixture where the spheres of species 1 are in a close packed configuration occupying a volume $V_{1}$ and the spheres of species 2 are in a closed packed configuration occupying a volume $V_{2}$ such that $\left.V_{2} \cap V_{1}=0\right)$. It gives a lower bound to the true maximum packing fraction.

In Cases I and III we have pure condensation as $\eta \rightarrow 0$. Case V display a pure condensation point at small but non-zero values of $\eta$. In Case II we find a pure demixing point at high $\eta$, for sufficiently large $\zeta$ in the same region where in Case IV we have a pure condensation point. The packing fraction of pure demixing for Case II can be easily calculated to be

$$
\eta=\frac{\langle\sigma\rangle\left\langle\sigma^{3}\right\rangle}{\left\langle\sigma^{4}\right\rangle}
$$

which turns out to be very close, albeit in general not coincident, with the packing fraction at which we find pure condensation in Case IV.

We remark that (both for mMSA and PY) the presence of an instability curve for the SHS model is entirely due to the stickiness, since in the HS limit $(\tau \rightarrow \infty)$ we have

$$
\lim _{\tau \rightarrow \infty} \operatorname{det}[\widehat{\mathbf{Q}}(0)]=\frac{1+2 \eta}{(1-\eta)^{2}}
$$

which is always a positive quantity. Eq. (98) can be derived from Eq. (54) by noticing that the contact values of the partial cavity functions $\bar{y}_{i j}$ must remain finite as $\tau \rightarrow \infty$. So the above statement is actually valid for any closure in which the partial direct correlation functions vanish beyond $\sigma_{i j}$. In particular it is valid for the mMSA and the PY [35] approximations. For other, thermodynamically more consistent closures, the statement is no longer true since phase separation has been observed for highly asymmetric HS binary mixtures [36]. 


\section{CONCLUSIONS}

In this work we have applied the method devised by Chen and Forstmann [5] to characterize the kind of thermodynamic instability to a number of carefully selected SHS binary systems. The crucial quantity turns out to be the Chen and Forstmann angle $\alpha$, see Eq. (41), on the spinodal: when $\alpha$ is close to 0 the instability is of the pure demixing type, whereas a value close to $\pm \pi / 2$ indicates a pure condensation instability.

The presence of adhesion between the spheres results in the existence of thermodynamic instabilities for the SHS model when treated within closures having the direct correlation functions vanishing beyond the hard core ranges, whereas it is known that the HS mixture within the same approximations do not show any instability [see Eq. (98)].

We have first considered the symmetric binary mixture in the mMSA (see Section IVA) and in the PY approximation (see Section IVB). This latter case was already considered by Chen and Forstmann for a different potential. We have found that when $\epsilon_{11} \leq \epsilon_{12}$ the instability is of pure condensation along the whole spinodal [see Fig. 2 and Eq. (63) for the mMSA, and Figs. 4 and 5 for the PY], while when $\epsilon_{11}>\epsilon_{12}$ a pure demixing spinodal appears at large packing fractions [see Fig. 3] and Eqs. (63) and (64) for the mMSA, and Figs. 4 and 5 for the PY], all within their respective limits of validity. This general behavior appears to be characteristic of symmetric binary mixtures, in the sense that it is observed in systems with pair potentials more "complex" than the SHS potential (hard spheres with Yukawa tails [37], square well [31], Lennard-Jones [38], etc.) which do not admit analytic solutions. The condensation and demixing lines are found to meet at a point in the mMSA, whereas they do not merge within the PY approximation.

Other two interesting examples can be treated in detail from an analytical point of view as discussed in Section Q We compared the spinodals and the angles $\alpha$ predicted by mMSA with those predicted by PY for a binary mixture with $\epsilon_{12}>0$ and $\epsilon_{11}=\epsilon_{22}=0$ (System A) and one with $\epsilon_{11}>0$ and $\epsilon_{12}=\epsilon_{22}=0$ (System B). Being the SHS interaction attractive, one should expect System A to present mainly condensation instabilities and System B mainly demixing instabilities. These choices for the $\epsilon_{i j}$ reduce the equations (53) for the contact values of the cavity functions in the PY approximation at most to a quadratic one, simplifying calculations considerably. We find that the spinodals predicted by the two approximations are very different both quantitatively and qualitatively [see Figs. 6] and [7. 
and Eqs. (84) and (85) for System A, and Fig. 10 and Eqs. (91) and (92) for System B]. Nonetheless the corresponding angles $\alpha$ do not depend on the closure, when this is chosen within the GPY large class containing mMSA and PY as particular cases. In agreement with our expectations, we find that the instabilities of System A are predominantly of the condensation type (see Fig. 8), while the ones of System B of the demixing type when $\zeta \simeq 1$ (see Fig. 11). For System B when we have a small number of large spheres of species 1, the demixing instability may be favored by both the osmotic depletion mechanism [32] and the stickiness between the large spheres (see Fig. 9).

In the more general case, the pair potential depends in general on 3 parameters: the ratio of the sphere diameters of the two species, $\zeta=\sigma_{2} / \sigma_{1}$, and two dimensionless parameters which measure the relative strength of surface adhesiveness, $\nu_{22}=\epsilon_{22} / \epsilon_{11}$ and $\nu_{12}=\epsilon_{12} / \epsilon_{11}$. A reduction occurs when the latters are connected to the former through plausible relationships $\epsilon_{i j}=\epsilon_{0} \mathcal{F}_{i j}\left(\sigma_{1}, \sigma_{2}\right)$. Following our previous work [26], we have considered five possible cases [see Section VI and Eq. (96)]. We find that four of the five cases exhibit very distinct types of instabilities (see Figs. 12 and 13): Cases I and III have the same angle $\alpha$, with pure condensation at $\eta \rightarrow 0$ and predominant demixing for $\eta>0$; Case $\mathrm{V}$ has a pure condensation instability point at low packing fractions; Case IV has a pure condensation instability point at high packing fractions provided that $\zeta$ is sufficiently large, whereas Case II has a pure demixing instability point under the same conditions.

It would be desirable to extend the present study in two respects. First it would be interesting to consider different, more sophisticated, closures, in view of our results on the two examples (denoted as System A and System B) where the angle $\alpha$ is shown to be independent of the particular closure within the GPY class, in spite of a large difference in the corresponding instability curves. Second, it would be nice to test the analytical predictions given in this work against numerical simulations, with a particular attention to what concerns the depletion mechanism. We plan to address both issues in a future work.

\section{APPENDIX A: THERMODYNAMIC RELATIONS FOR THE ELEMENTS OF THE $M$ MATRIX}

In this appendix we gather together some well known relationships between thermodynamic quantities and the results obtained in the main text. The Ashcroft-Langreth partial 
structure factors [39] of an homogeneous and isotropic $p$-component mixture are related to the partial total correlation functions as

$$
S_{i j}(k)=\delta_{i j}+\rho \sqrt{x_{i} x_{j}} \tilde{h}_{i j}(k)
$$

where $x_{i}=\left\langle N_{i}\right\rangle /\langle N\rangle$ is the molar fraction of particles of species $i$ and $\rho$ the total density of the mixture. From the normalization condition for the partial pair distribution functions of the grand canonical ensemble follows

$$
S_{i j}(0)=\sqrt{\frac{x_{i}}{x_{j}}}\left(\frac{\left\langle N_{i} N_{j}\right\rangle-\left\langle N_{i}\right\rangle\left\langle N_{j}\right\rangle}{\left\langle N_{i}\right\rangle}\right),
$$

The matrix $\tilde{\mathbf{A}}$, defined in Eq. (13) of the text, is related to the structure factors by

$$
S_{i j}(k)=\left[\tilde{\mathbf{A}}^{-1}\right]_{i j}(k) .
$$

We now relate composition fluctuations to thermodynamic quantities. The grand partition function is

$$
e^{-\beta \Omega}=\sum_{N_{1}, \ldots, N_{p}=0}^{\infty} e^{\beta\left[\sum_{i=1}^{p} N_{i} \mu_{i}-A\left(T, V,\left\{N_{i}\right\}\right)\right]},
$$

where $A\left(T, V,\left\{N_{i}\right\}\right)$ is the Helmholtz free energy of a member of the grand canonical ensemble with given number of particles of each species, and the chemical potentials $\left\{\mu_{i}\right\}$ are to be determined from the average number of particles of each species

$$
\left\langle N_{i}\right\rangle=\sum_{N_{1}, \ldots, N_{p}=0}^{\infty} N_{i} e^{\beta\left[\Omega+\sum_{i=1}^{p} N_{i} \mu_{i}-A\left(T, V,\left\{N_{i}\right\}\right)\right]} .
$$

We immediately find

$$
\left(\frac{\partial \Omega}{\partial \mu_{i}}\right)_{T, V,\left\{\mu_{\bar{\imath}}\right\}}=-\left\langle N_{i}\right\rangle
$$

and

$$
\begin{aligned}
\frac{1}{\beta}\left(\frac{\partial N_{i}}{\partial \mu_{j}}\right)_{T, V,\left\{\mu_{\bar{\jmath}}\right\}} & =\left\langle N_{i}\right\rangle\left(\frac{\partial \Omega}{\partial \mu_{j}}\right)_{T, V,\left\{\mu_{\bar{\jmath}}\right\}}+\left\langle N_{i} N_{j}\right\rangle \\
& =\left\langle N_{i} N_{j}\right\rangle-\left\langle N_{i}\right\rangle\left\langle N_{j}\right\rangle=\sqrt{x_{i} x_{j}} S_{i j}(0)\langle N\rangle,
\end{aligned}
$$

where the index $\bar{\imath}$ denotes all species different from $i$. Since the thermodynamic derivatives $\left(\partial N_{i} / \partial \mu_{j}\right)_{T, V,\left\{\mu_{\bar{j}}\right\}}$ are the elements of the inverse of the matrix whose elements are $\left(\partial \mu_{i} / \partial N_{j}\right)_{T, V,\left\{N_{\bar{j}}\right\}}$ we can invert the above relation to read

$$
\begin{aligned}
\beta\left(\frac{\partial \mu_{i}}{\partial N_{j}}\right)_{T, V,\left\{N_{\bar{\jmath}}\right\}} & =\frac{1}{\langle N\rangle \sqrt{x_{i} x_{j}}}\left[\mathbf{S}^{-1}\right]_{i j}(0) \\
& =\frac{1}{V \rho \sqrt{x_{i} x_{j}}} \tilde{A}_{i j}(0)
\end{aligned}
$$


where we indicated with $\mathbf{S}$ the matrix whose elements are the partial structure factors.

We now define the partial volumes as

$$
v_{i}=\left(\frac{\partial V}{\partial N_{i}}\right)_{T, P,\left\{N_{\bar{\imath}}\right\}} .
$$

Since the total volume is an homogeneous function of order one in the extensive variables we must have

$$
\sum_{i=1}^{p} N_{i} v_{i}=V
$$

since the Gibbs free energy $G=G\left(T, P,\left\{N_{i}\right\}\right)$ is an homogeneous function of order one in the extensive variables we must have

$$
\sum_{i=1}^{p} N_{i} \mu_{i}=G
$$

so in particular the chemical potentials will be homogeneous functions of order zero in the variables $\left\{N_{i}\right\}$, we can then write $\mu_{i}=\mu_{i}\left(T, P,\left\{\left\{N_{i}\right\}\right\}\right)$ where with the symbol $\left\{\left\{N_{i}\right\}\right\}$ we mean that the variables $\left\{N_{i}\right\}$ can appear only as ratios. We also find

$$
\left(\frac{\partial \mu_{i}}{\partial N_{j}}\right)_{T, V,\left\{N_{\bar{j}}\right\}}=\left(\frac{\partial \mu_{i}}{\partial N_{j}}\right)_{T, P,\left\{\left\{N_{\bar{\jmath}}\right\}\right\}}+\frac{v_{i} v_{j}}{V \chi_{T}},
$$

where $\chi_{T}$ is the isothermal compressibility

$$
\chi_{T}=-\frac{1}{V}\left(\frac{\partial V}{\partial P}\right)_{T,\left\{N_{i}\right\}} .
$$

Notice also that taking the partial derivative of Eq. (A11) with respect to $N_{j}$ at constant $T, P$, and $\left\{N_{\bar{\jmath}}\right\}$ we find the following Gibbs-Duhem relation

$$
\sum_{i=1}^{p} N_{i}\left(\frac{\partial \mu_{i}}{\partial N_{j}}\right)_{T, P,\left\{\left\{N_{\bar{\jmath}}\right\}\right\}}=0
$$

We want now find thermodynamic relations for the matrix elements $M_{\rho \rho}, M_{x x}$, and $M_{\rho x}$ of the binary mixture. We will do the calculation explicitly for $M_{\rho \rho}$ and quote the final result for the other two elements. So from Eq. (23) we find for $M_{\rho \rho}$

$$
\begin{aligned}
M_{\rho \rho} & =x_{1}\left(1-\rho x_{1} \tilde{c}_{11}\right)+x_{2}\left(1-\rho x_{2} \tilde{c}_{22}\right)-\rho x_{1} x_{2}\left(\tilde{c}_{12}+\tilde{c}_{21}\right) \\
& =V \rho \beta \sum_{i, j=1}^{2} x_{i} x_{j}\left(\frac{\partial \mu_{i}}{\partial N_{j}}\right)_{T, V,\left\{N_{\bar{j}}\right\}} \\
& =\frac{\rho \beta}{\chi_{T}} \sum_{i, j=1}^{2} x_{i} x_{j} v_{i} v_{j} \\
& =\frac{\chi_{T}^{0}}{\chi_{T}}
\end{aligned}
$$


where $\chi_{T}^{0}=\beta / \rho$ is the isothermal compressibility of the ideal gas, in the second equality Eqs. (13) and (A8) were used, in the third equality we used Eqs. (A12) and (A14) and in the last equality Eq. (A10). For $M_{\rho x}$ we find

$$
M_{\rho x}=\sqrt{x_{1} x_{2}} \delta \frac{\chi_{T}^{0}}{\chi_{T}}
$$

where

$$
\delta \equiv \rho\left(v_{1}-v_{2}\right)=\frac{1}{V}\left(\frac{\partial V}{\partial x_{1}}\right)_{T, P, N}
$$

and for $M_{x x}$

$$
M_{x x}=x_{1} x_{2} \delta^{2} \frac{\chi_{T}^{0}}{\chi_{T}}+x_{1} x_{2} \frac{\chi_{T}^{0}}{V}\left(\frac{\partial^{2} G}{\partial x_{1}^{2}}\right)_{T, P, N} .
$$

The determinant factorizes

$$
\operatorname{det}(\mathbf{M})=\operatorname{det}(\tilde{\mathbf{A}})=[\operatorname{det}(\widehat{\mathbf{Q}})]^{2}=x_{1} x_{2} \frac{\left(\chi_{T}^{0}\right)^{2}}{\chi_{T} V}\left(\frac{\partial^{2} G}{\partial x_{1}^{2}}\right)_{T, P, N},
$$

thus yielding Eq. (26) in the main text.

\section{ACKNOWLEDGMENTS}

This work was supported by the Italian MIUR (PRIN-COFIN 2004/2005).

[1] J. S. Rowlinson, Liquids and Liquid Mixtures (Butterworths, London, 1969), 2nd ed.

[2] C. H. P. Lupis, Chemical Thermodynamics of Materials (North-Holland, Dordrecht, 1983).

[3] D. Gazzillo, Mol. Phys. 83, 1171 (1994).

[4] D. Gazzillo, Mol. Phys. 84, 303 (1995).

[5] X. S. Chen and F. Forstmann, J. Chem. Phys. 97, 3696 (1992).

[6] C. P. Ursenbach and G. N. Patey, J. Chem. Phys. 100, 3827 (1994).

[7] A. B. Bhatia and D. E. Thornton, Phys. Rev. B 2, 3004 (1970).

[8] R. J. Baxter, J. Chem. Phys. 49, 2770 (1968).

[9] R. J. Baxter, in Physical Chemistry, an Advanced Treatise, vol. 8A, ed. D. Henderson (Academic Press, New York, 1971) ch. 4.

[10] R. O. Watts, D. Henderson, and R. J. Baxter, Advan. Chem. Phys. 21, 421 (1971).

[11] R. J. Baxter, J. Chem. Phys. 52, 4559 (1970). 
[12] B. Barboy, Chem. Phys. 11, 357 (1975).

[13] J. W. Perram and E. R. Smith, Chem. Phys. Lett. 35, 138 (1975).

[14] B. Barboy and R. Tenne, Chem. Phys. 38, 369 (1979).

[15] G. Stell, J. Stat. Phys. 63, 1203 (1991).

[16] C. Robertus, W. H. Philipse, J. G. H. Joosten, and Y. K. Levine, J. Chem. Phys. 90, 4482 (1989).

[17] S. H. Chen, J. Rouch, F. Sciortino, and P. Tartaglia, J. Phys.:Condens. Matter 6, 10855 (1994).

[18] H. Löwen, Phys. Rep. 237, 249 (1994).

[19] G. Nägele, Phys. Rep. 272, 215 (1996).

[20] D. Gazzillo and A. Giacometti, J. Chem. Phys. 113, 9837 (2000).

[21] D. Gazzillo and A. Giacometti, J. Chem. Phys. 120, 4742 (2004).

[22] Note that the mMSA solution for the Baxter factor function of the SHS binary model can also be obtained [40] from the model of a mixture of hard spheres interacting through Yukawa potentials in the MSA 41] upon taking the limit of a vanishing screening length.

[23] M. H. G. M. Penders and A. Vrij, Physica A 173, 532 (1991).

[24] C. Regnaut, S. Amokrane, and Y. Heno, J. Chem. Phys. 102, 6230 (1995).

[25] S. Amokrane and C. Regnaut, J. Chem. Phys. 106, 376 (1997).

[26] R. Fantoni, D. Gazzillo, and A. Giacometti, J. Chem. Phys. 122, 034901 (2005).

[27] J. M. Caillol, J. Phys. A-Math. Gen. 35, 4189 (2002).

[28] L. D. Landau and E. M. Lifshitz, Statistical Physics (Butterworth-Heinemann, 2001), 3rd ed., part 1.

[29] Note that in the mMSA, unlike in the PY, $y_{i j}\left(\sigma_{i j}^{+}\right)$differs from $y_{i j}\left(R_{i j}^{+}\right)$even in the sticky limit, in view of the fact that $y_{i j}(r)$ is not continuous at $R_{i j}$. This feature allows to obtain the correct HS result in the limit of no adhesion.

[30] Our Eq. (54) corrects Eq. (60) of Barboy and Tenne 14] where a sign is misprinted. Note also that these authors use the symbol $d_{i j}$ in place of our $\sigma_{i j}$ and $\tau_{i j}^{B T}=6 \tau_{i j}$ where the superscript $B T$ indicates that the symbol is the one used by Barboy and Tenne.

[31] N. B. Wilding, F. Schmid, and P. Nielaba, Phys. Rev. E 58, 2201 (1998).

[32] S. Asakura and F. Oosawa, J. Chem. Phys. 22, 1255 (1954).

[33] J. L. Barrat and J. P. Hansen, Basic Concepts for Simple and Complex Liquids (Cambridge, 
2003).

[34] P. Sollich (2005), after the submission of this work it has been brought to our attention [P. Sollich, private communication] that Cases I, III, and V have a pair interaction potential [see Eq. (45)] which might include many-body effects due to the presence of the averages. This is bacause, for instance, the interaction between two particles of species 1 depends also on the diameter of the particles of species 2 . As a complement to this observation, we also note that as the diameter of one species of particles, say $\sigma_{1} \rightarrow 0$, vanishes, then -according to Eq. (45)- those pointwise particles are non-interacting among themselves thus requiring $K_{11} \rightarrow 0$ as well. This constraint would rule out Cases I and III.

[35] J. L. Lebowitz and J. S. Rowlinson, J. Chem. Phys. 41, 133 (1964).

[36] T. Biben and J.-P. Hansen, Phys. Rev. Lett. 66, 2215 (1991).

[37] C. Jȩdrzejek, J. Konior, and M. Streszewski, Phys. Rev. A 35, 1226 (1987).

[38] O. Antonevych, F. Forstmann, and E. Diaz-Herrera, Phys. Rev. E 65, 061504 (2002).

[39] N. W. Ashcroft and D. C. Langreth, Phys. Rev. 156, 685 (1967).

[40] D. Gazzillo and A. Giacometti, Mol. Phys. 101, 2171 (2003).

[41] L. Blum and J. S. Høye, J. Stat. Phys. 19, 317 (1978). 


\section{LIST OF FIGURES}

Fig. 1 Schematic representation of the two orthonormal vectors $\mathbf{z}_{ \pm}$defined in Eq. (32) and of the angle $\alpha$ defined in Eq. (411) when $\alpha \in[0, \pi / 2]$. When $\alpha \in[-\pi / 2,0]$ the angle shown in the Fig. corresponds to $|\alpha|$ and $\delta \bar{x}$ to $-\delta \bar{x}$.

Fig. 2 Spinodal line (continuous curve) for the symmetric mixture in the mMSA with $\nu_{12}=2$. The kind of instability is of pure condensation along the whole spinodal.

Fig. 3 Spinodal line (continuous curve) for the symmetric mixture in the mMSA with $\nu_{12}=$ $2 / 3$. In this case the instability is of pure condensation for $\eta<\eta_{\rho x}$ along $\tau^{*}=\tau_{\rho}^{*}$ and of pure demixing for $\eta>\eta_{\rho x}$ along $\tau^{*}=\tau_{x}^{*}$.

Fig. 4 Spinodal line for the symmetric mixture in the PY approximation with $\nu_{12}=2$ in the top panel and with $\nu_{12}=2 / 3$ in the bottom panel. At $\nu_{12}=2$ the instability is of pure condensation along $\tau^{*}=\left(\tau_{\rho}^{*}\right)_{i}, i=a, b$ and of pure demixing along $\tau^{*}=\left(\tau_{x}^{*}\right)_{i}$, $i=a, b$. The zeroes labeled $c$ are unphysical. The gaps between the curves $\tau^{*}=\left(\tau_{\rho}^{*}\right)_{i}$ are numerical artifacts. At $\nu_{12}=2 / 3$ there is the appearance of a pure demixing spinodal at high $\eta$ which does not cross the pure condensation one. For reference we also plot in both panels the spinodal of the one component system $\tau_{o c}^{*}$ [see Eq. (76)] which is physical only for $\eta>\eta_{c}=(3 \sqrt{2}-4) / 2=0.1213 \ldots$.

Fig. 5 Same as Fig. [ with $\nu_{12}=1 / 0.9$ in the top panel and $\nu_{12}=1 / 1.1$ in the bottom panel. In this last case the expected line of pure demixing would start at $\eta>\eta_{0}=0.7404 \ldots$ in the unphysical range of densities.

Fig. 6 For System A the mMSA spinodal [see Eq. [84)] for $\zeta=2$ and three different values of $x_{1}$.

Fig. 7 For System A the PY spinodal [see Eq. (85)] under the same conditions considered in Fig. [6]

Fig. 8 Behavior of the angle $\alpha$ of Eq. (41) predicted by the mMSA and PY for System A when $x_{1}=0.75, \zeta=2$. In this case the PY spinodal has no solutions when $\eta>0.03227 \ldots$. In the inset we show the region of $\eta$ were the PY spinodal exists. Note that here and in the following $\cos \alpha$ rather than the angle $\alpha$ itself is depicted for visual convenience. 
Fig. 9 In System B, when we have a small number of large particles of species 1, the demixing instability [see Eq. (94)] should be favored by the osmotic depletion mechanism, since the small spheres interact through a HS potential both among themselves and with the big spheres.

Fig. 10 For System B the spinodals predicted by mMSA [thick lines, see Eq. (91)] and the ones predicted by PY [thin lines, see Eq. (92)] for $\zeta=1$ at three different values of $x_{1}$. The physically meaningful PY spinodals are those lying above the "existence" lines in accord with condition (93).

Fig. 11 For System B behavior of the angle $\alpha$ of Eq. (41) predicted by mMSA and PY for $x_{1}=0.91$ and $\zeta=1$ in the bottom panel (in this case the PY spinodal has loss of solution for $\eta<\eta_{e} \approx 0.1248 \ldots$..) and $\zeta=2$ in the top panel (in this case the PY spinodal has loss of solution for $\left.\eta<\eta_{e} \approx 0.1614 \ldots\right)$.

Fig. 12 Behavior of the angle $\alpha$ of Eq. (41) for Cases I, II, III, IV, and V when $x_{1}=1 / 2$ and $\zeta=3 / 2$.

Fig. 13 Behavior of the angle $\alpha$ of Eq. (41) for Cases I, II, III, IV, and V when $x_{1}=1 / 2$ and $\zeta=5$. 


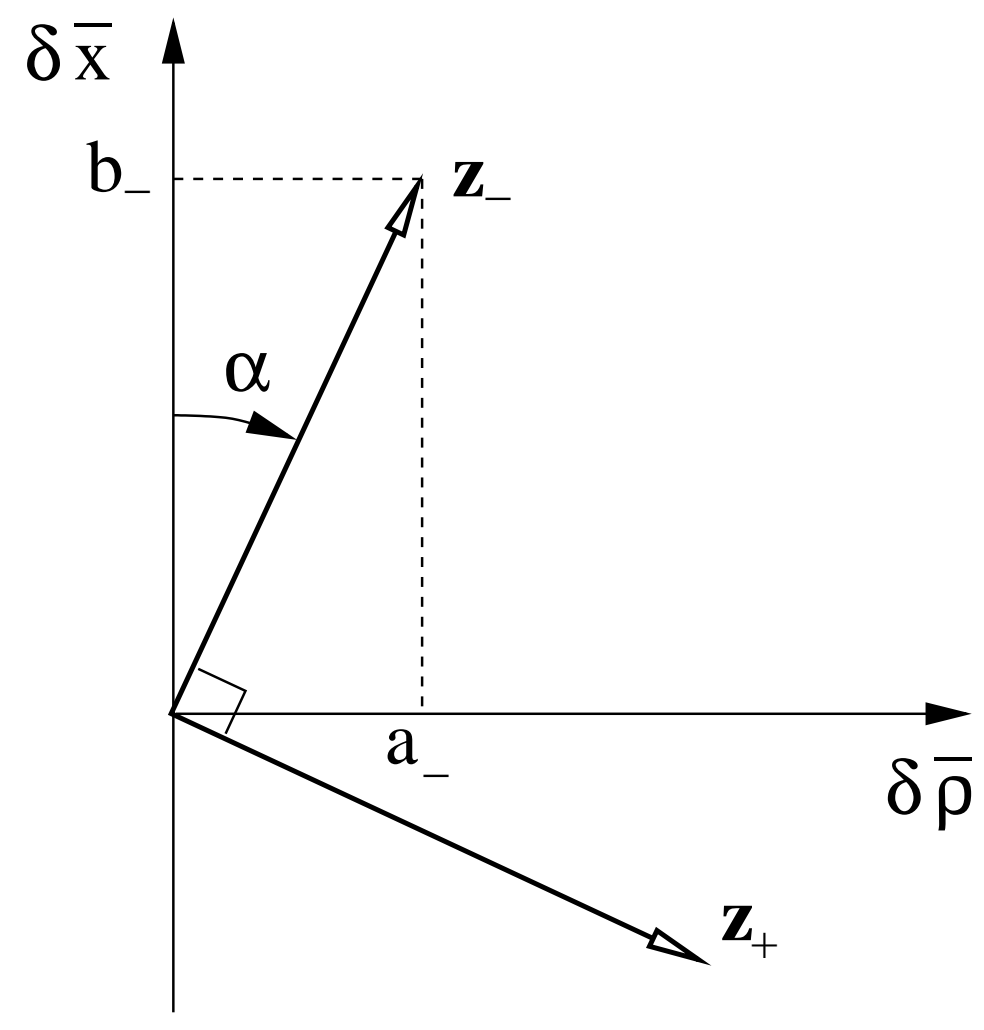

FIG. 1: Schematic representation of the two orthonormal vectors $\mathbf{z}_{ \pm}$defined in Eq. (32) and of the angle $\alpha$ defined in Eq. (41) when $\alpha \in[0, \pi / 2]$. When $\alpha \in[-\pi / 2,0]$ the angle shown in the Fig. corresponds to $|\alpha|$ and $\delta \bar{x}$ to $-\delta \bar{x}$. 


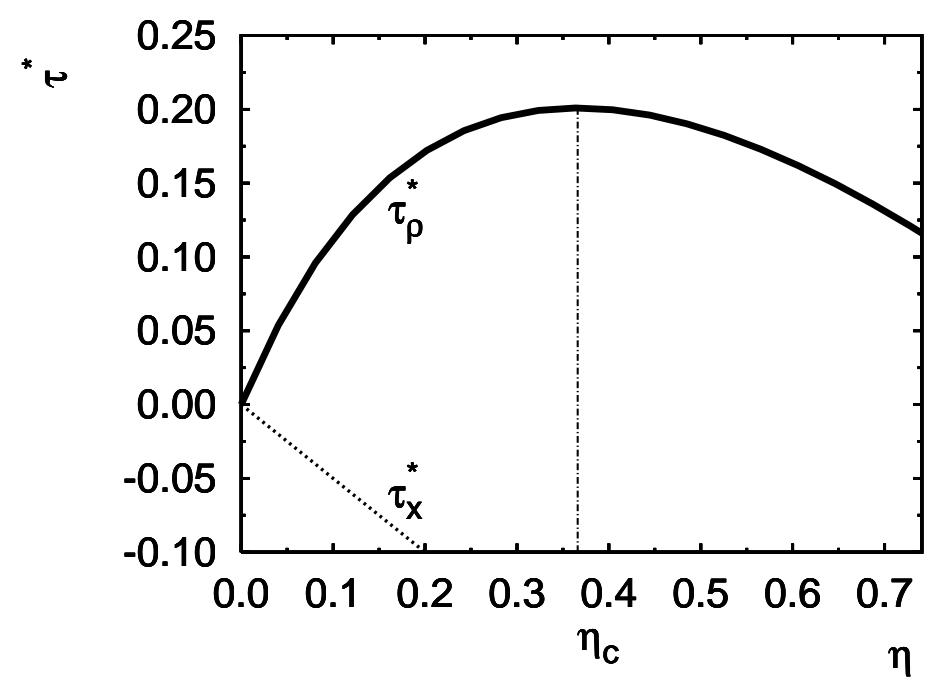

FIG. 2: Spinodal line (continuous curve) for the symmetric mixture in the mMSA with $\nu_{12}=2$. The kind of instability is of pure condensation along the whole spinodal.

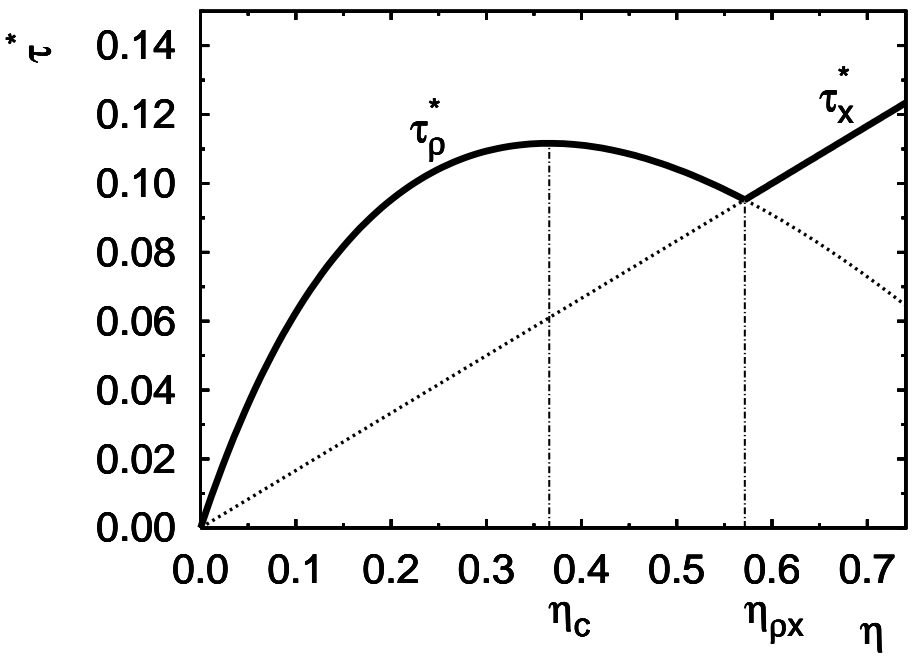

FIG. 3: Spinodal line (continuous curve) for the symmetric mixture in the mMSA with $\nu_{12}=2 / 3$. In this case the instability is of pure condensation for $\eta<\eta_{\rho x}$ along $\tau^{*}=\tau_{\rho}^{*}$ and of pure demixing for $\eta>\eta_{\rho x}$ along $\tau^{*}=\tau_{x}^{*}$. 


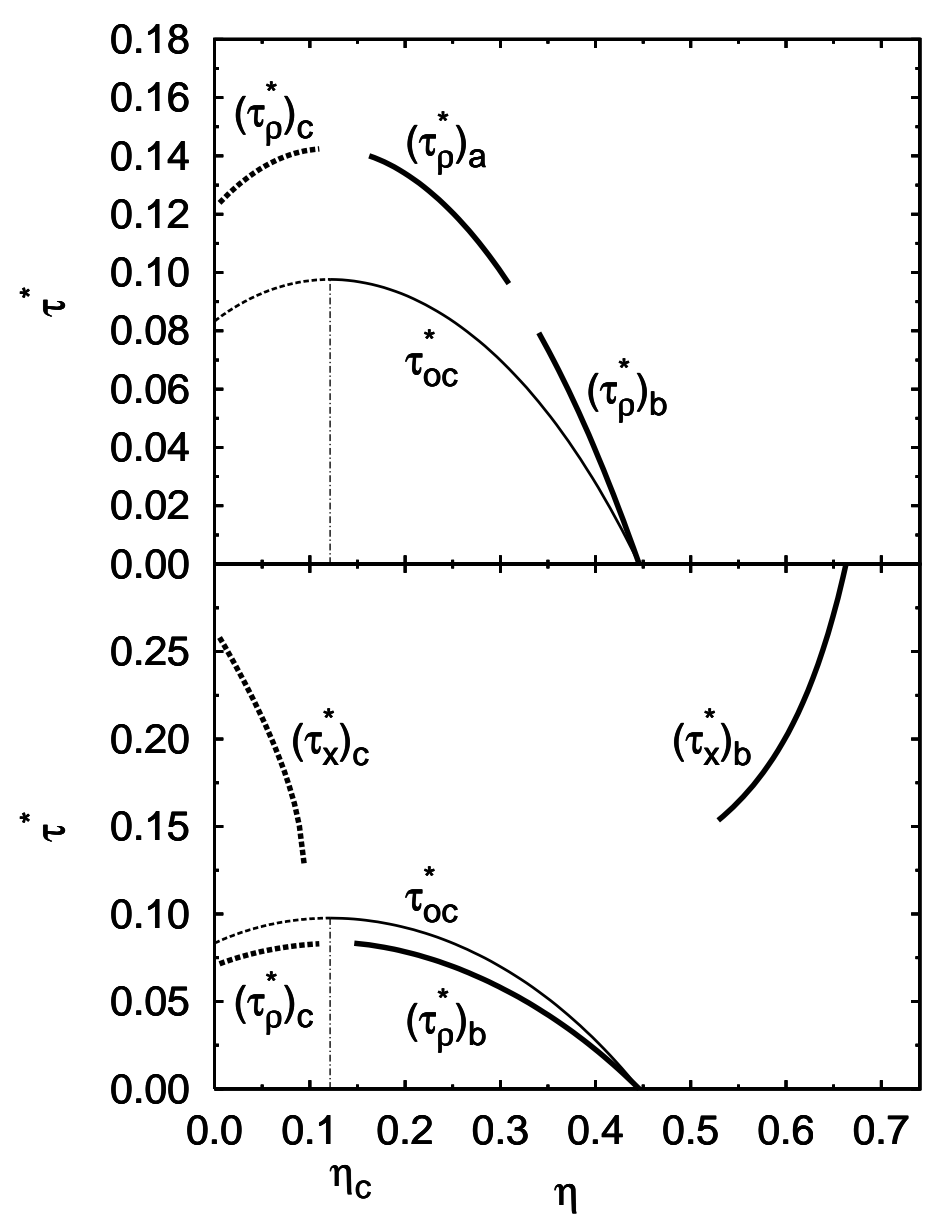

FIG. 4: Spinodal line for the symmetric mixture in the PY approximation with $\nu_{12}=2$ in the top panel and with $\nu_{12}=2 / 3$ in the bottom panel. At $\nu_{12}=2$ the instability is of pure condensation along $\tau^{*}=\left(\tau_{\rho}^{*}\right)_{i}, i=a, b$ and of pure demixing along $\tau^{*}=\left(\tau_{x}^{*}\right)_{i}, i=a, b$. The zeroes labeled $c$ are unphysical. The gaps between the curves $\tau^{*}=\left(\tau_{\rho}^{*}\right)_{i}$ are numerical artifacts. At $\nu_{12}=2 / 3$ there is the appearance of a pure demixing spinodal at high $\eta$ which does not cross the pure condensation one. For reference we also plot in both panels the spinodal of the one component system $\tau_{o c}^{*}$ [see Eq. (76) ] which is physical only for $\eta>\eta_{c}=(3 \sqrt{2}-4) / 2=0.1213 \ldots$. 


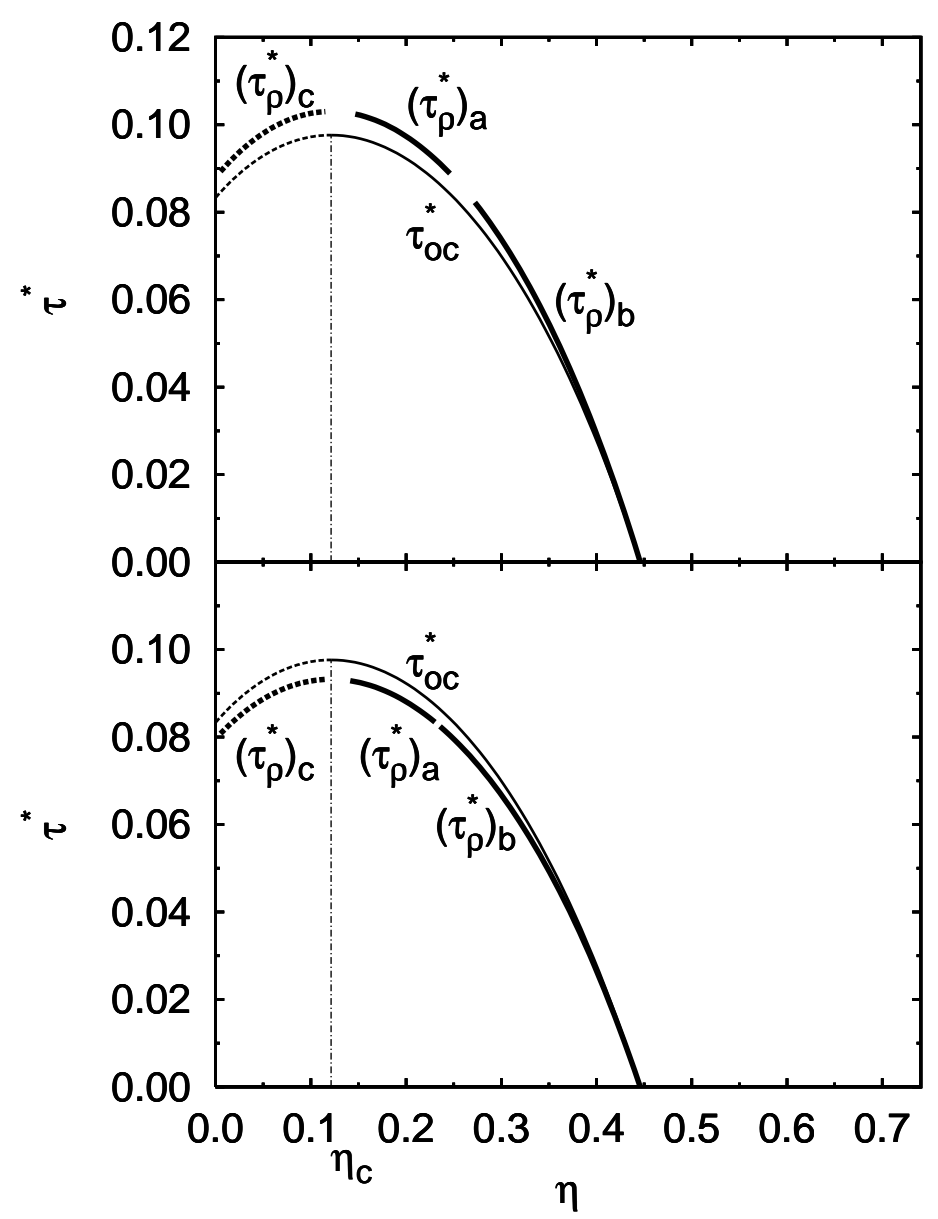

FIG. 5: Same as Fig. 4 with $\nu_{12}=1 / 0.9$ in the top panel and $\nu_{12}=1 / 1.1$ in the bottom panel. In this last case the expected line of pure demixing would start at $\eta>\eta_{0}=0.7404 \ldots$ in the unphysical range of densities. 


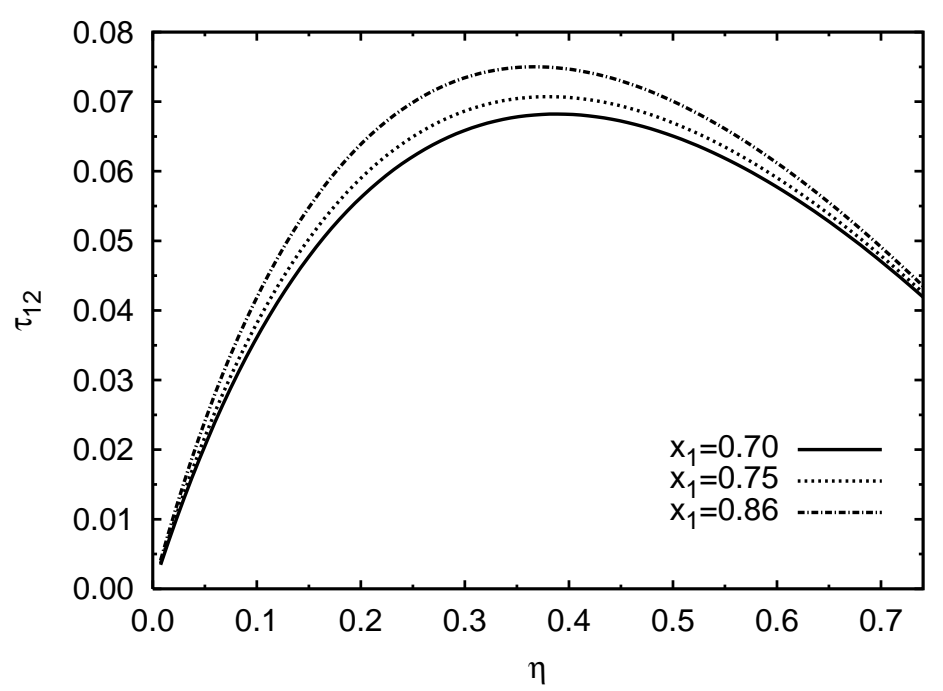

FIG. 6: For System A the mMSA spinodal [see Eq. [84)] for $\zeta=2$ and three different values of $x_{1}$.

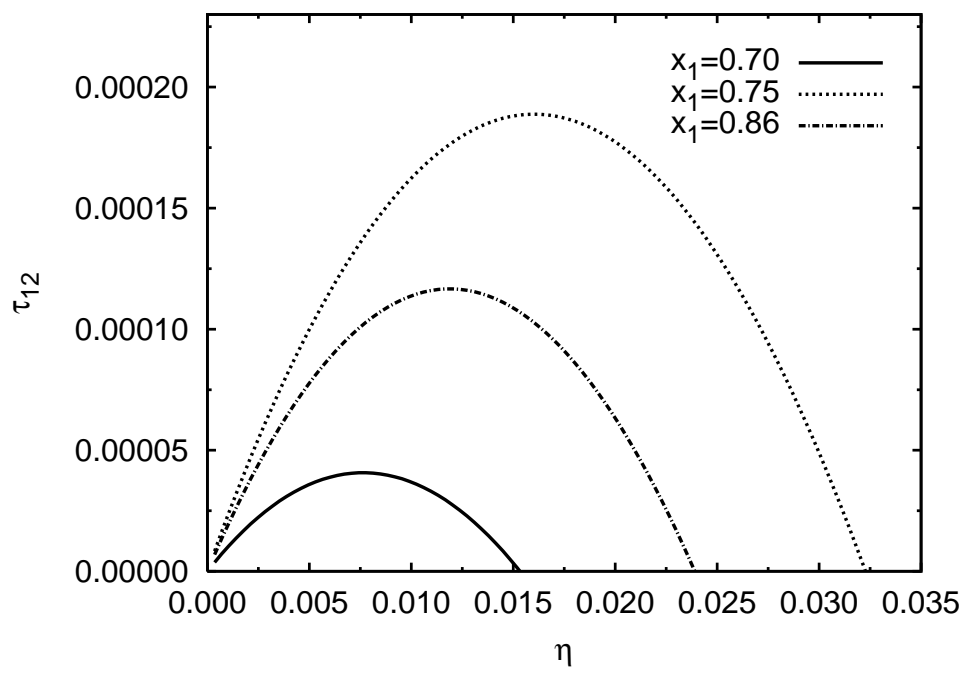

FIG. 7: For System A the PY spinodal [see Eq. [85 )] under the same conditions considered in Fig. 6 , 


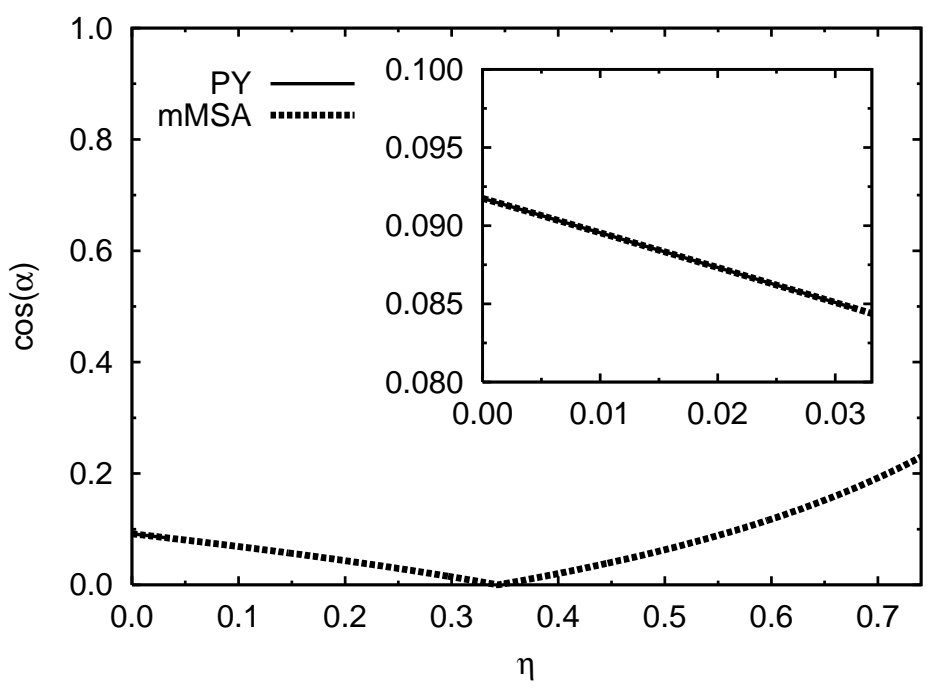

FIG. 8: Behavior of the angle $\alpha$ of Eq. (41) predicted by the mMSA and PY for System A when $x_{1}=0.75, \zeta=2$. In this case the PY spinodal has no solutions when $\eta>0.03227 \ldots$ In the inset we show the region of $\eta$ were the PY spinodal exists. Note that here and in the following $\cos \alpha$ rather than the angle $\alpha$ itself is depicted for visual convenience.

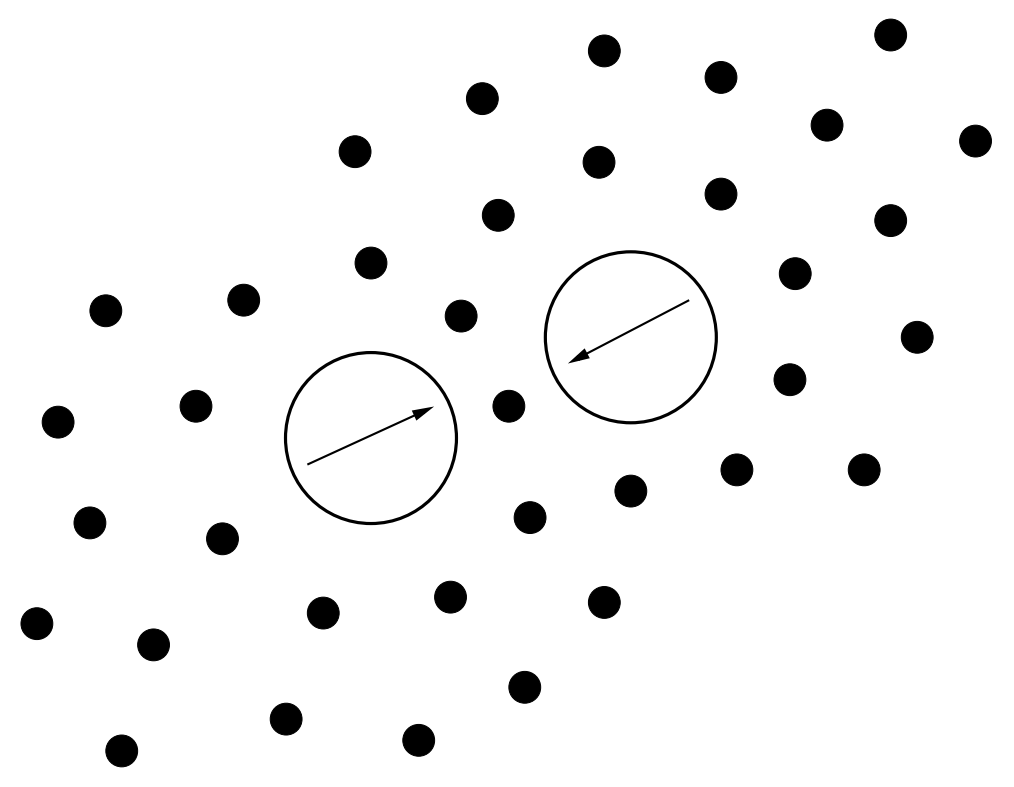

FIG. 9: In System B, when we have a small number of large particles of species 1, the demixing instability [see Eq. (94)] should be favored by the osmotic depletion mechanism, since the small spheres interact through a HS potential both among themselves and with the big spheres. 


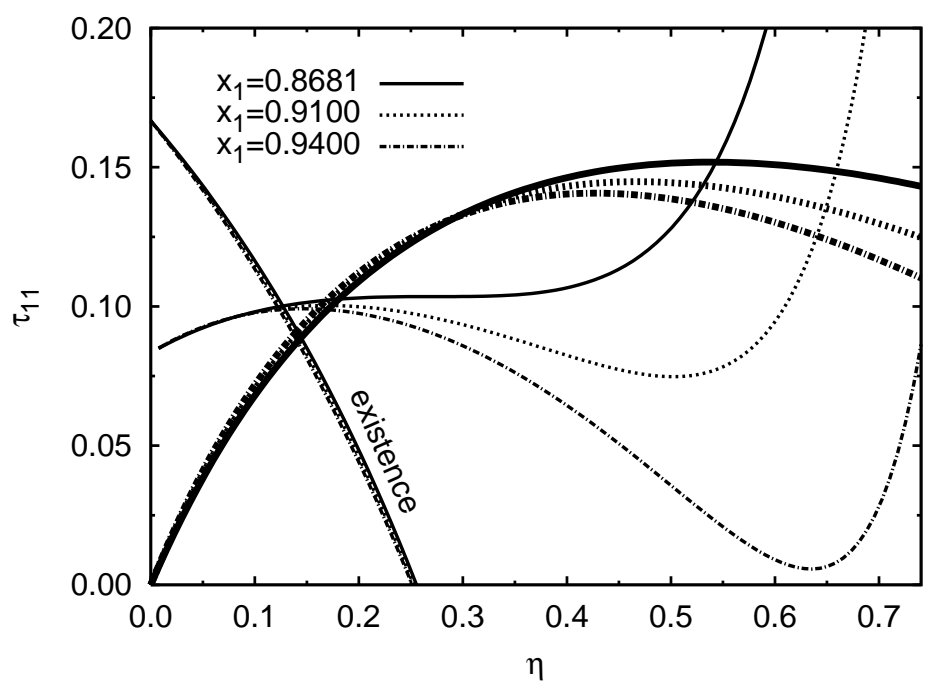

FIG. 10: For System B the spinodals predicted by mMSA [thick lines, see Eq. (91)] and the ones predicted by PY [thin lines, see Eq. (92)] for $\zeta=1$ at three different values of $x_{1}$. The physically meaningful PY spinodals are those lying above the "existence" lines in accord with condition (93). 


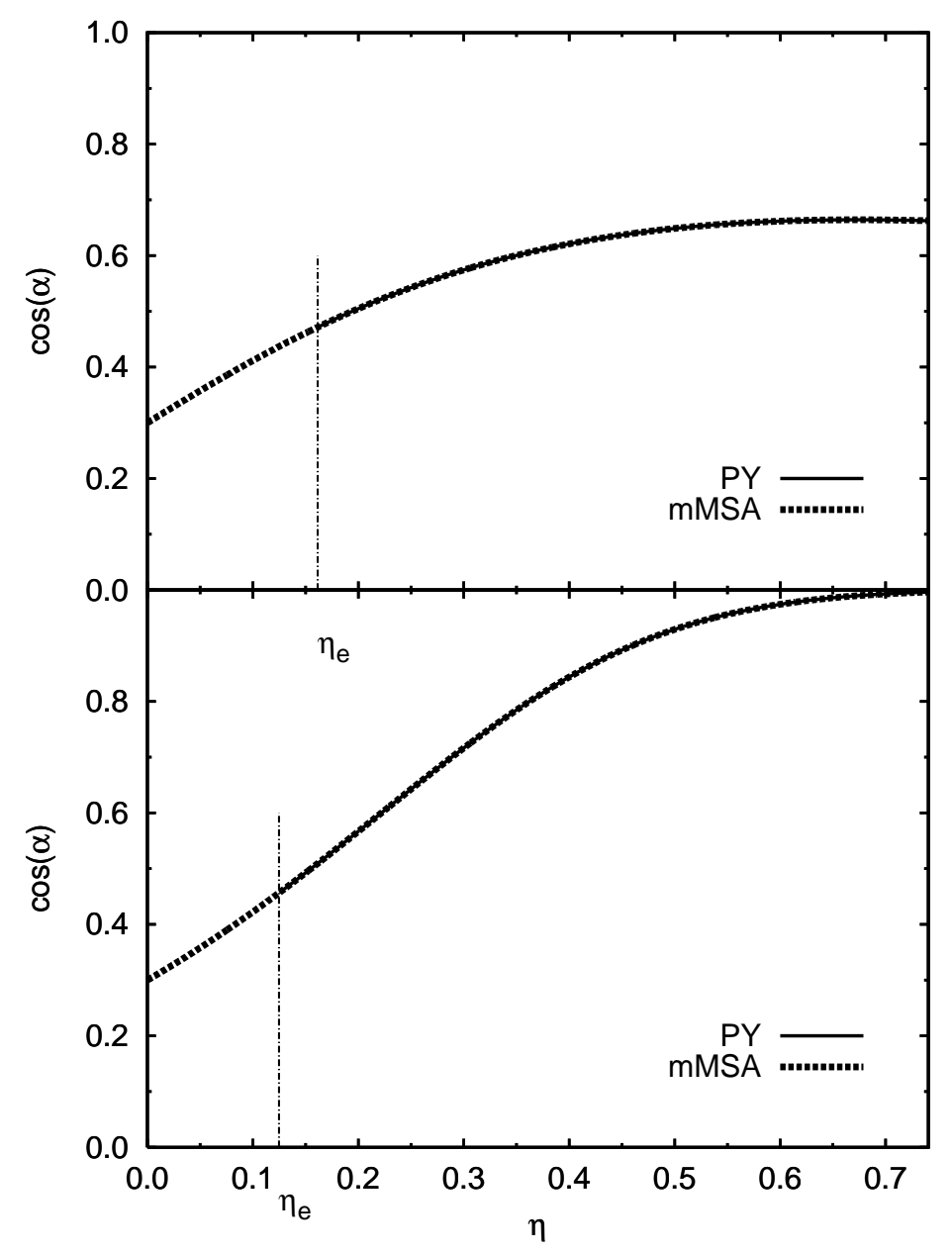

FIG. 11: For System B behavior of the angle $\alpha$ of Eq. (41) predicted by mMSA and PY for $x_{1}=0.91$ and $\zeta=1$ in the bottom panel (in this case the PY spinodal has loss of solution for $\eta<\eta_{e} \approx 0.1248 \ldots$.) and $\zeta=2$ in the top panel (in this case the PY spinodal has loss of solution for $\left.\eta<\eta_{e} \approx 0.1614 \ldots\right)$. 


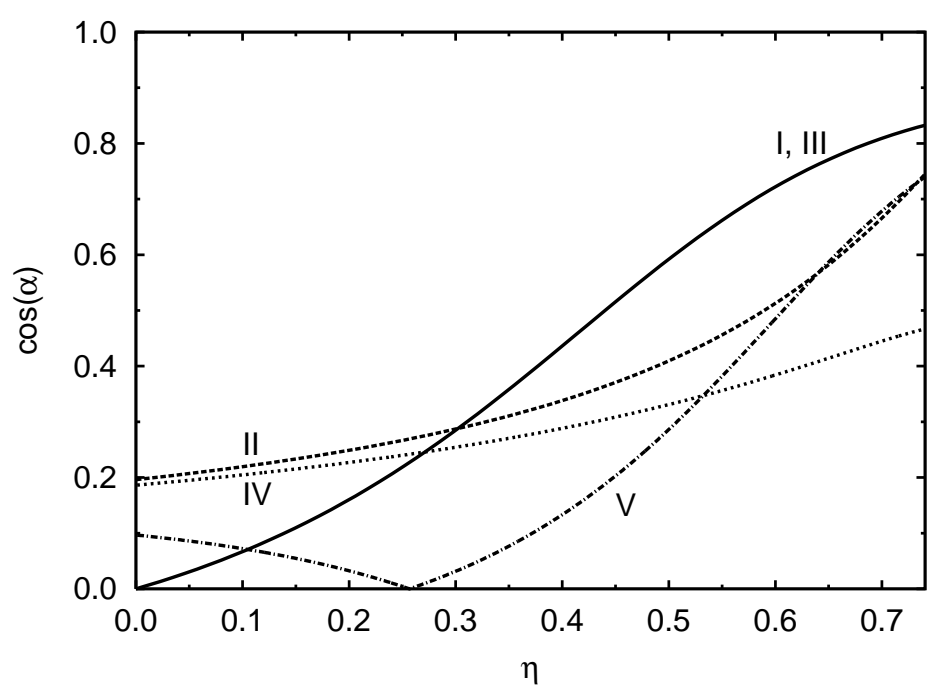

FIG. 12: Behavior of the angle $\alpha$ of Eq. (41) for Cases I, II, III, IV, and V when $x_{1}=1 / 2$ and $\zeta=3 / 2$.

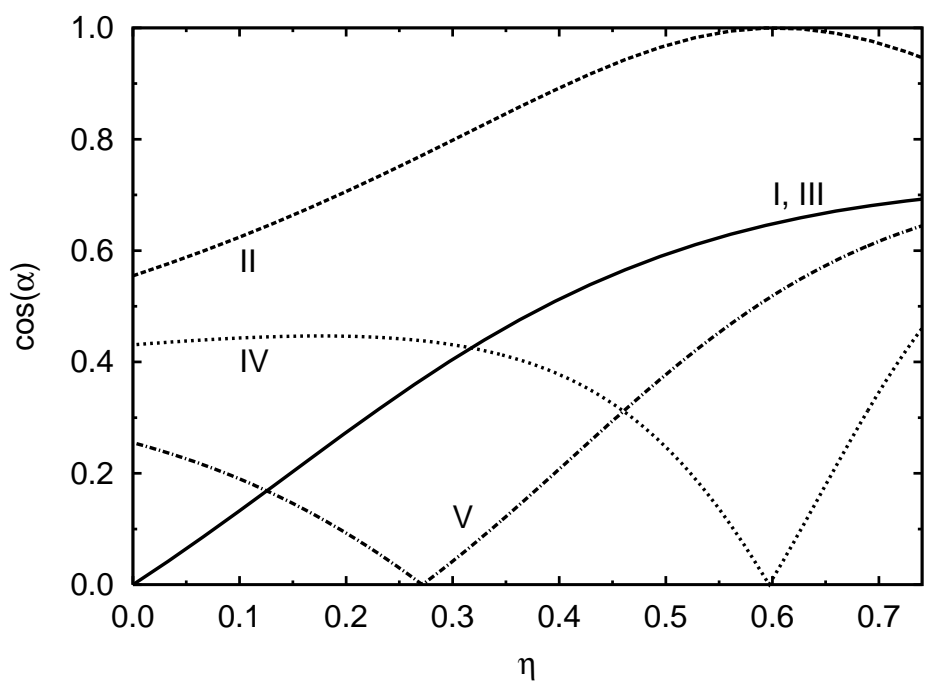

FIG. 13: Behavior of the angle $\alpha$ of Eq. (41) for Cases I, II, III, IV, and V when $x_{1}=1 / 2$ and $\zeta=5$. 